
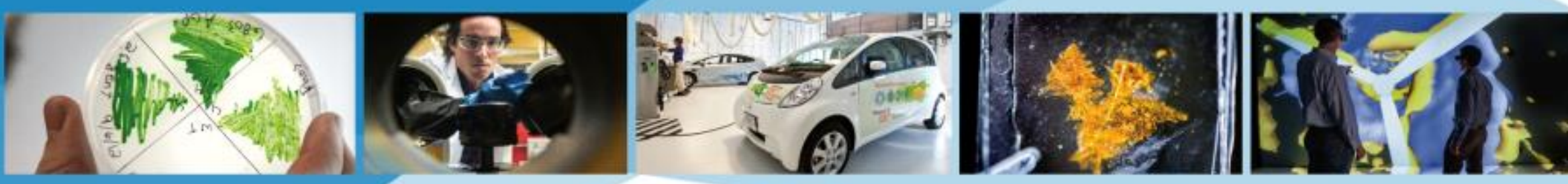

\title{
Quantitative Microstructure Characterization of a NMC Electrode
}

François L.E. Usseglio-Viretta $^{\mathrm{a}}$

Kandler Smith ${ }^{\mathrm{a}}$

a National Renewable Energy Laboratory

Presented at the $231^{\text {st }}$ ECS Meeting

New Orleans, Louisiana

May 30, 2017

NREL/PR-5400-68741

NREL is a national laboratory of the U.S. Department of Energy, Office of Energy Efficiency and Renewable Energy, operated by the Alliance for Sustainable Energy, LLC. 


\section{Outline}

Characterization of NMC (already segmented) microstructures

with a focus on numerical methods, bias/assumption, RVE size, and voxel $\underline{\text { size dependence }}$

- Introducing original "level of details" parameters

- Volume fractions and connectivity

- Particle size

$\circ$ Calendaring effect

- Correlate RVE size with standard deviation

○ Results from different methods compared

- Tortuosity factor

○ Voxel size and RVE analysis (including isotropy)

- Introducing original two-step homogenization method

$\circ$ Calendaring effect

- Spatial variation along electrode thickness.

Surface area results are in the annexes slides 


\section{Microstructures investigated}

$>$ Un-calendared (UC) \& Calendared (C) positive NMC $\left(\mathrm{LiNi}_{1 / 3} \mathrm{Mn}_{1 / 3} \mathrm{Co}_{1 / 3} \mathrm{O}_{2}\right)$ electrode, with different composition of active material (wt 90\%, 92\%, 94\%, and 96\%).

Raw (X-ray tomographic microscopy) and binarized image available online (open-source data) on the ETH (Swiss Federal Institute of Technology Zurich) website.

Ebner et al. Adv. Energy Mater. 2013, 3, 845-850

$>$ Secondary phase has not been identified (considered as pore)

$>$ Total of 16 Volumes

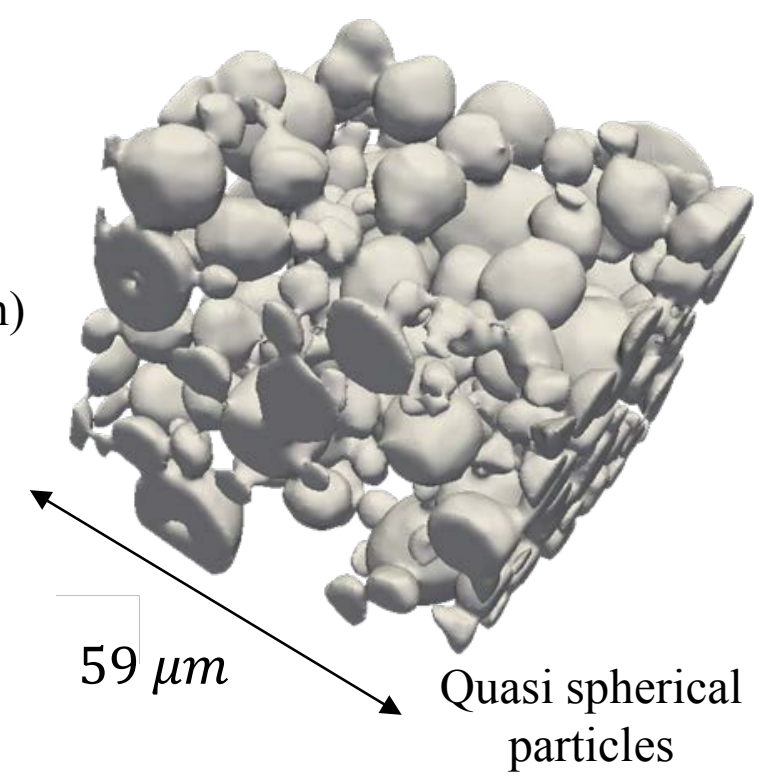

Analyzed domain size: $328.9 \times 328.9 \times A \mu m^{3}$

Equivalent domain size: $B \times B \times B \mu m^{3}$

\begin{tabular}{ccccc}
\hline $\begin{array}{c}\text { Composition of the } \\
\text { active material (\%) }\end{array}$ & $\begin{array}{c}\text { Un-calendared } \\
\text { electrode }\end{array}$ & $\begin{array}{c}\text { Calendared electrode } \\
(300 \text { bar })\end{array}$ & $\begin{array}{c}\text { Calendared electrode } \\
(600 \text { bar })\end{array}$ & $\begin{array}{c}\text { Calendared electrode } \\
(2,000 \text { bar })\end{array}$ \\
\hline 90 & $\mathbf{5 0 . 3 ( 1 7 6 )}$ & $\mathbf{5 2 . 2 ( 1 7 8 )}$ & $47.7(173)$ & $57.0(183)$ \\
92 & $45.9(171)$ & $77.3(203)$ & $58.8(185)$ & $61.1(188)$ \\
94 & $65.9(192)$ & $78.8(204)$ & $74.0(200)$ & $64.8(191)$ \\
96 & $98.1(220)$ & $94.7(217)$ & $101.0(222)$ & $87.7(212)$
\end{tabular}

Voxel size: $370 \times 370 \times 370 \mathrm{~nm}^{3}$ 


\section{Level of details of a microstructure}

\section{Introducing parameters dedicated to evaluate the image quality}

$\underset{\substack{\text { Edge } \\ \text { vatio }}}{\text { ratio }}=\left\{\begin{array}{c}1-\frac{\text { Edge "volume" }}{\text { Phase volume }} \\ 1-\frac{\sum \square}{\sum \boldsymbol{\square}+\sum \square}\end{array}\right.$

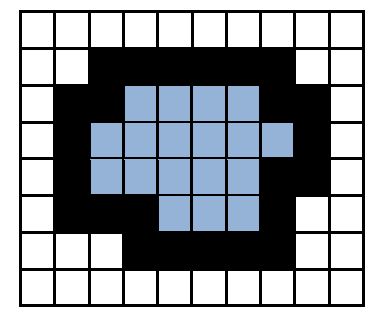

$\leq$

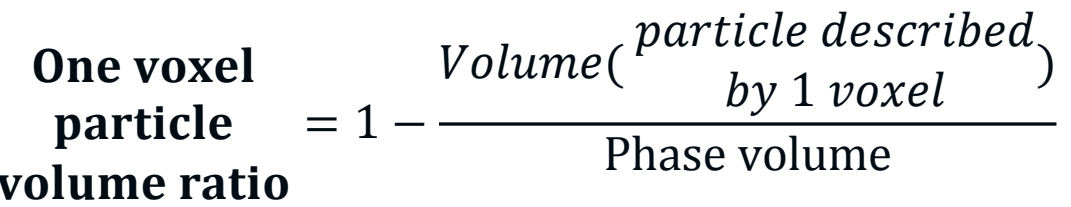

Will increase if smooth interface
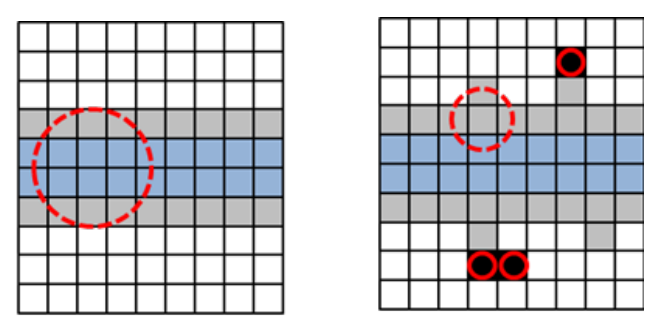

$>$ Based upon edge detection

$>$ Easy to interpretate: values ranges from

0 (poor) to 1 (ideal)

$>$ Ideal to compare different data set image

quality and different obs. techniques 


\section{Level of details of a microstructure}

\section{Introducing parameters dedicated to evaluate the image quality}

$\underset{\text { volume }}{\text { ratio }}=\left\{\begin{array}{c}1-\frac{\text { Edge "volume" }}{\text { Phase volume }} \\ 1-\frac{\sum \mathbf{q}}{\sum \mathbf{q}+\sum \mathbf{\square}}\end{array}\right.$
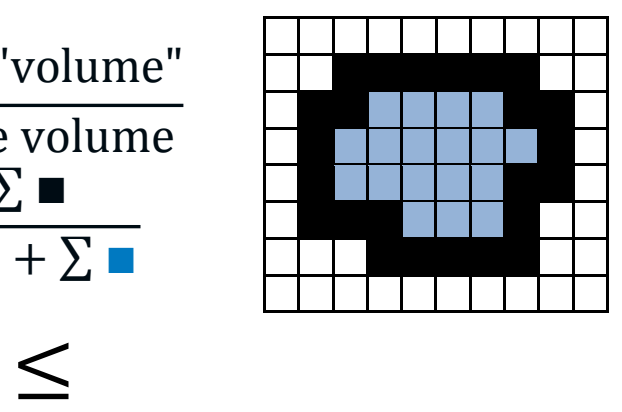

\section{One voxel particle $=1-$ volume ratio}

Will increase if smooth interface
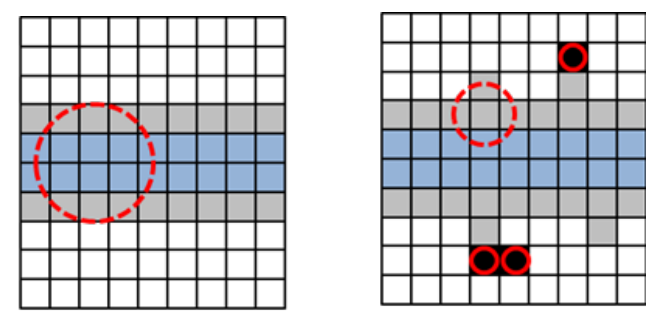

$>$ Based upon edge detection

$>$ Easy to interpretate: values ranges from 0 (poor) to 1 (ideal)

$>$ Ideal to compare different data set image quality and different obs. techniques

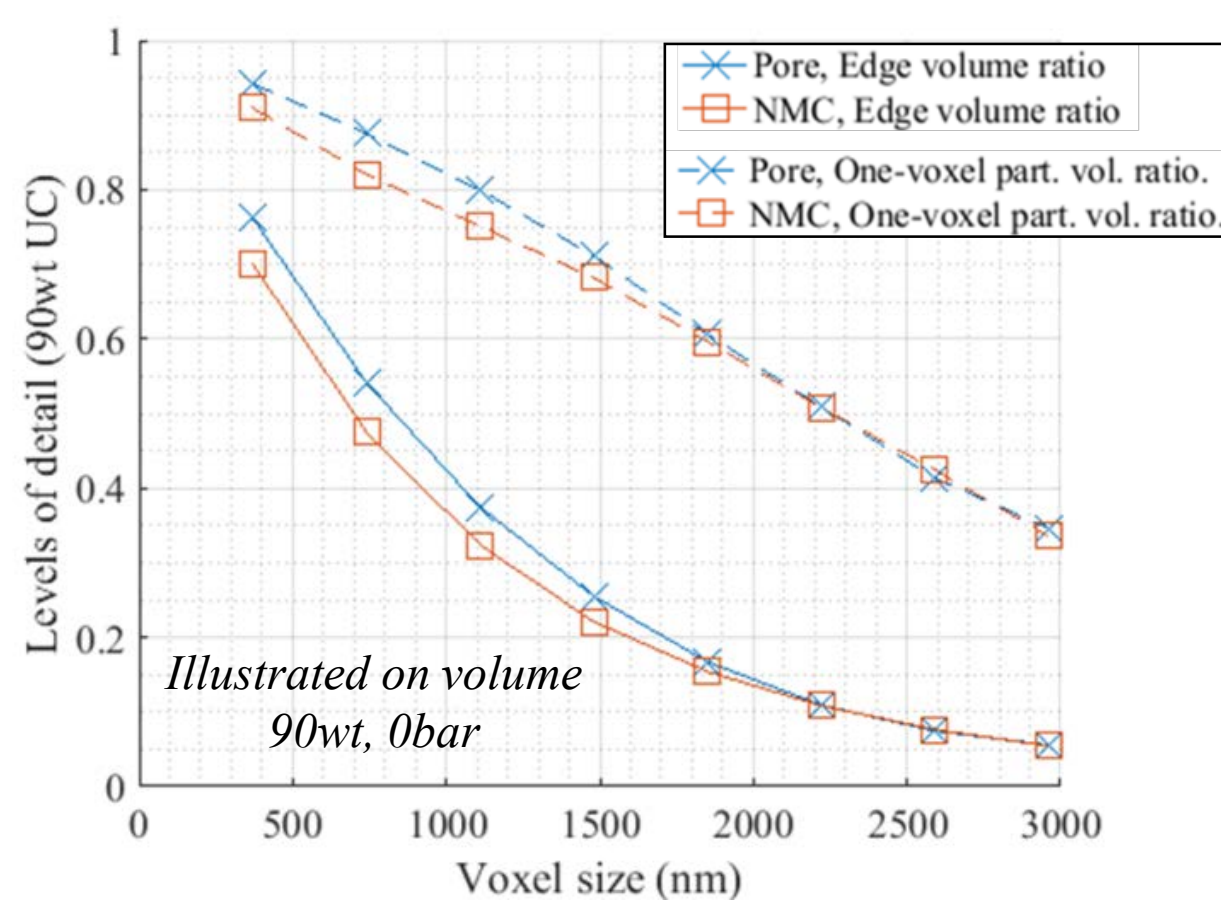

- As expected, level of details are degrading monotonously with image resolution.

- High level of details have been achieved by Ebner group.

- Both phases exhibits similar values (2-phase data-set). 


\section{Volume fraction \& connectivity}

\section{Results obtained on the 16 volumes ( 4 calendaring $X 4$ active material composition)}
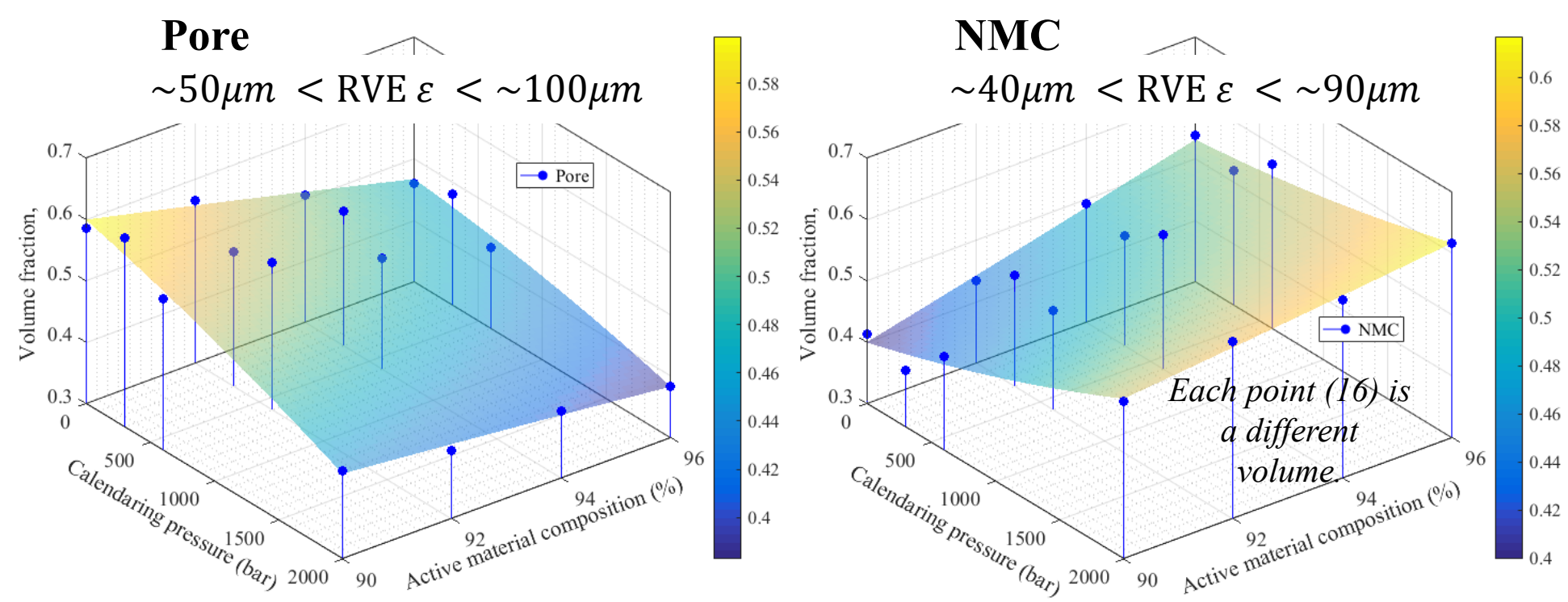

Porosity is $\searrow$ when active material composition is $\nearrow$ (because secondary phase has been identified as pore) and when calendaring pressure is $\nearrow$ 


\section{Volume fraction \& connectivity}

Edge effect induce a loss of percolation on small domains, especially for NMC

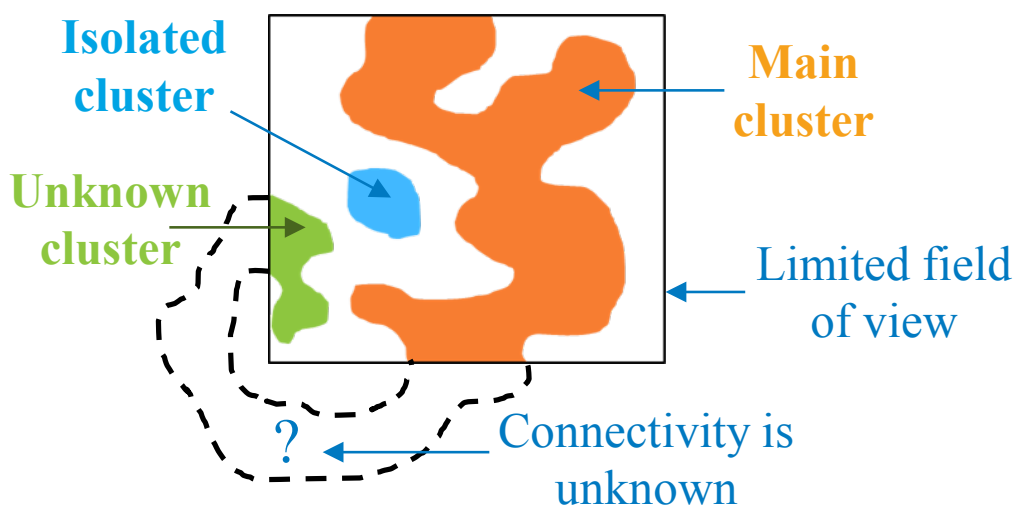

J. Joos et al., Journal of Power Sources 246, 819-830 (2014).
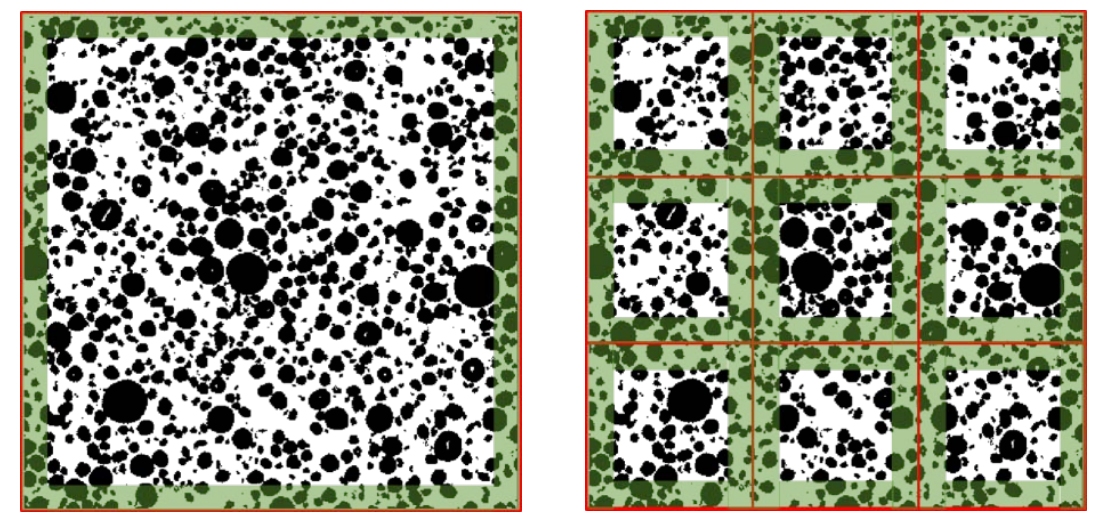

Unknown clusters are accumulating on

subdomains $\rightarrow$ global amount of unknown voxel

is increasing. 


\section{Volume fraction \& connectivity}

\section{Edge effect induce a loss of percolation on small domains, especially for NMC}
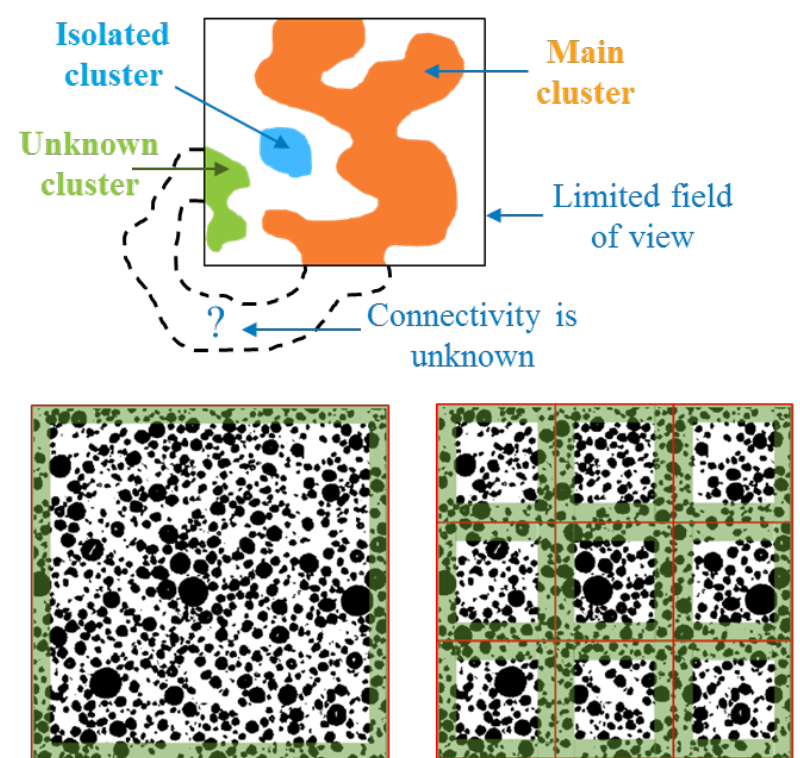

Unknown clusters are accumulating on subdomains $\rightarrow$ global amount of unknown voxel is increasing.
Illustrated on NMC, $90 \mathrm{wt}, 0$ bar
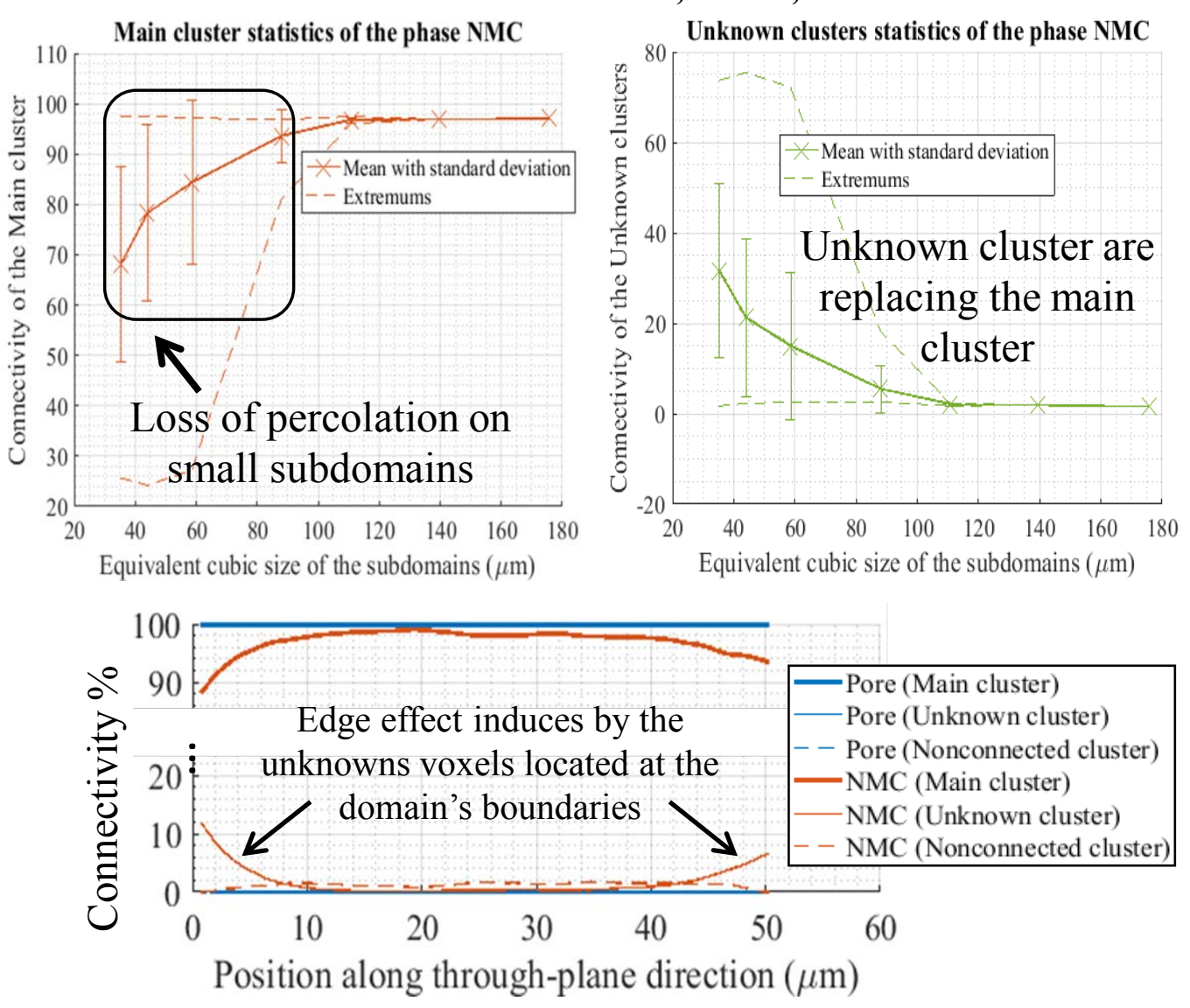

NMC small subdomains suffer from loss of percolation due to this edge effect. Pore is untouched. 


\section{Volume fraction \& connectivity}

This edge affects mainly the solid phase when calendaring pressure is not applied, as a result RVE for the connectivity is much more higher for the NMC

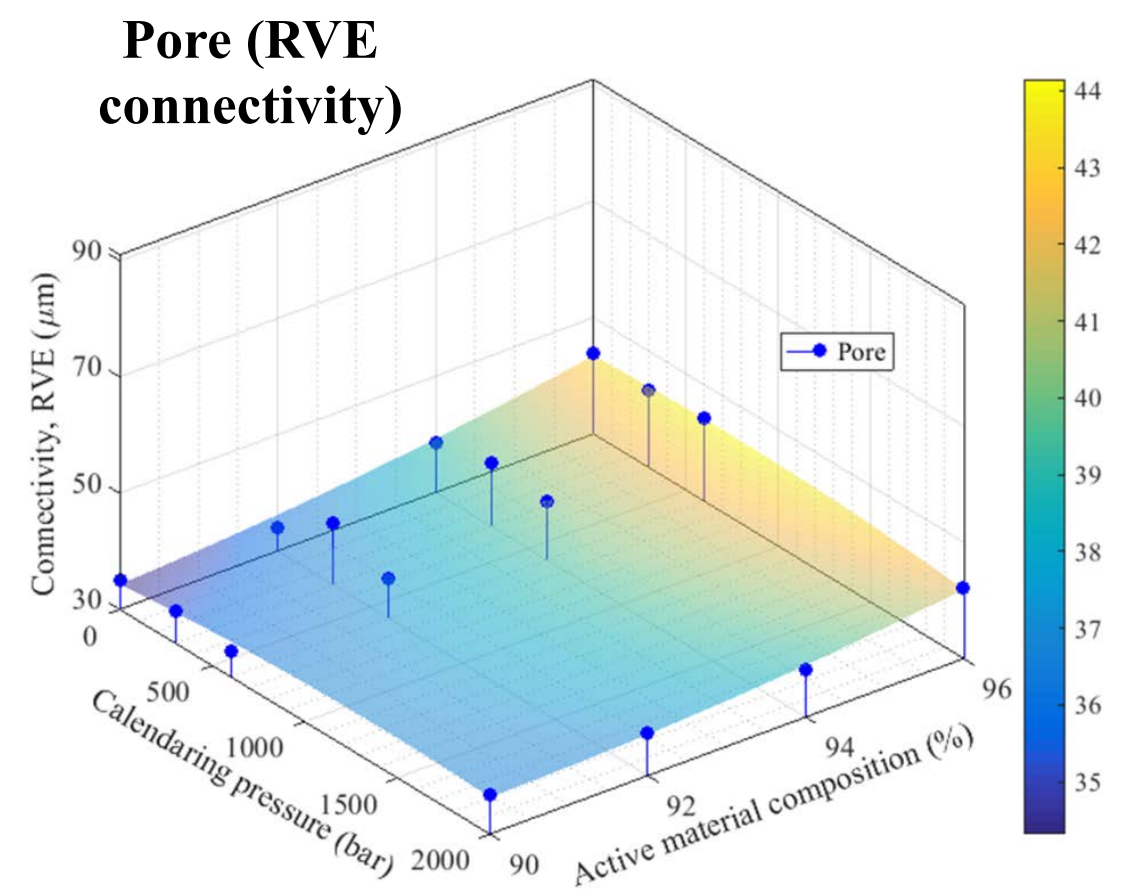

Pore exhibits excellent connectivity even for small volume (RVE is $<45 \mu \mathrm{m}$ for all volumes)

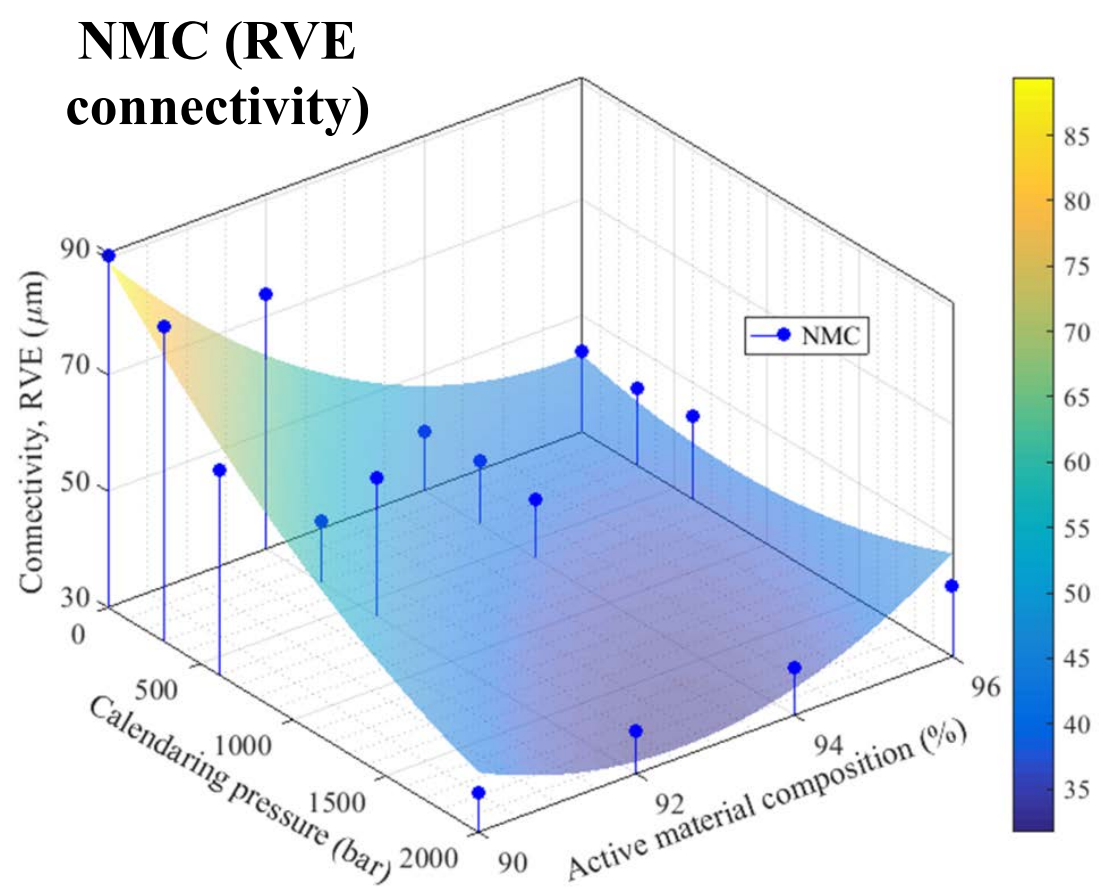

On the contrary, NMC need a larger volume to get connected. High calendaring pressure and active material composition reduce the RVE. 


\section{Particle size: spherical assumption (C-PSD)}

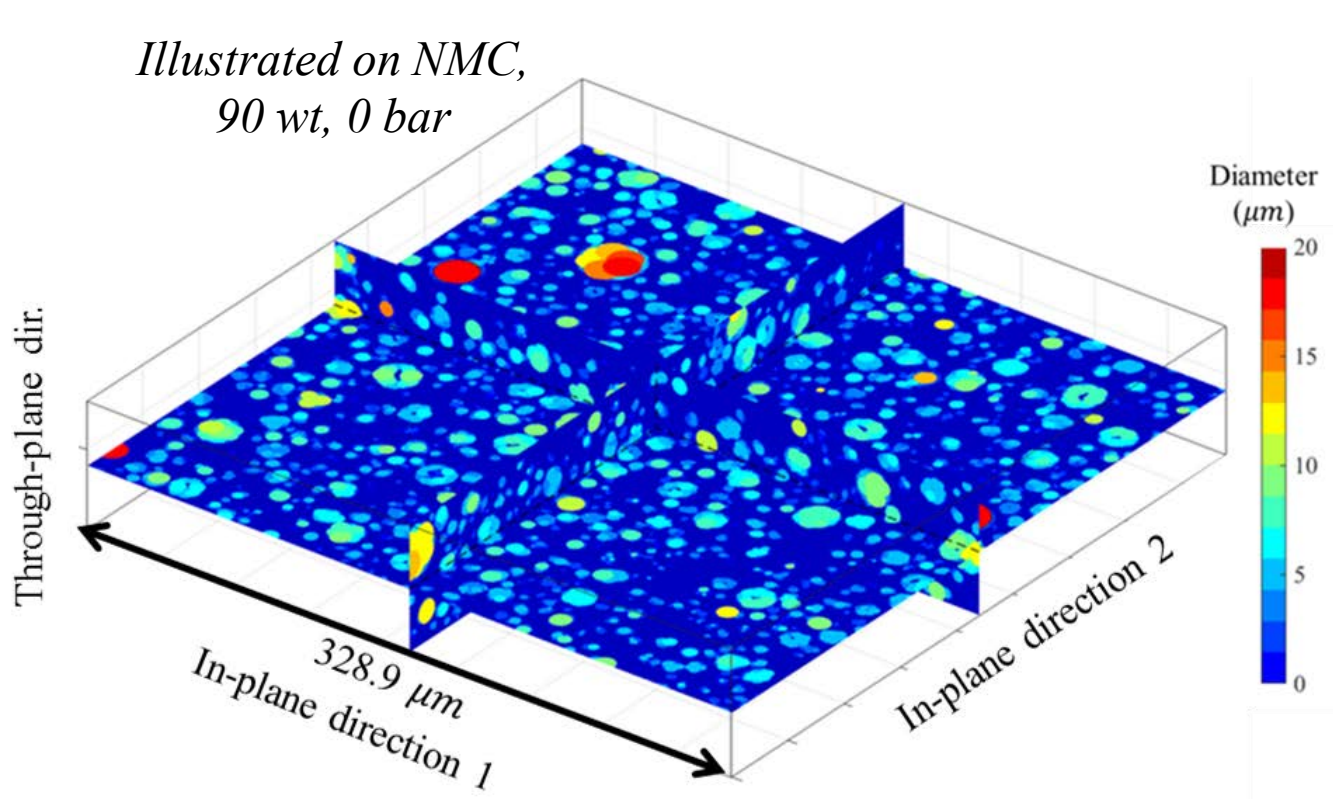

Voxels are attributed to the largest sphere that contains them.

L. D. Gelb and K. E. Gubbins, Langmuir 1999, 15, 305-308

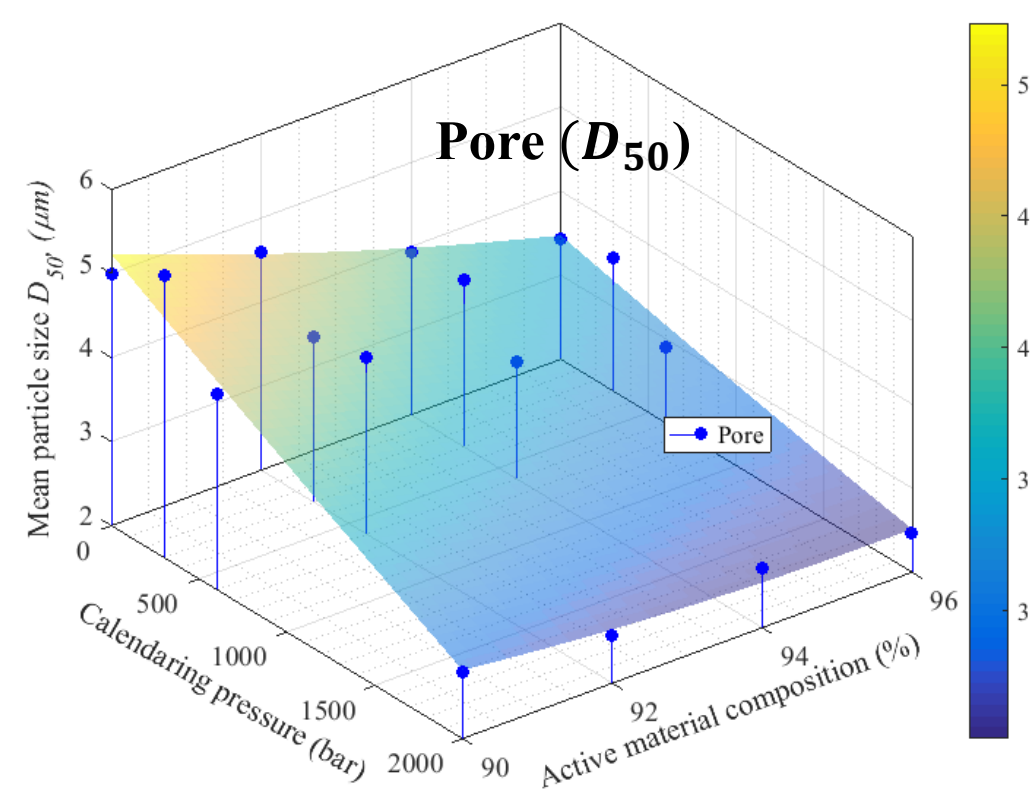

Calendaring pressure significantly reduces the pore size. Slight effect on the NMC

\section{Underestimation of the size distribution is \\ expected due to this particle shape assumption}

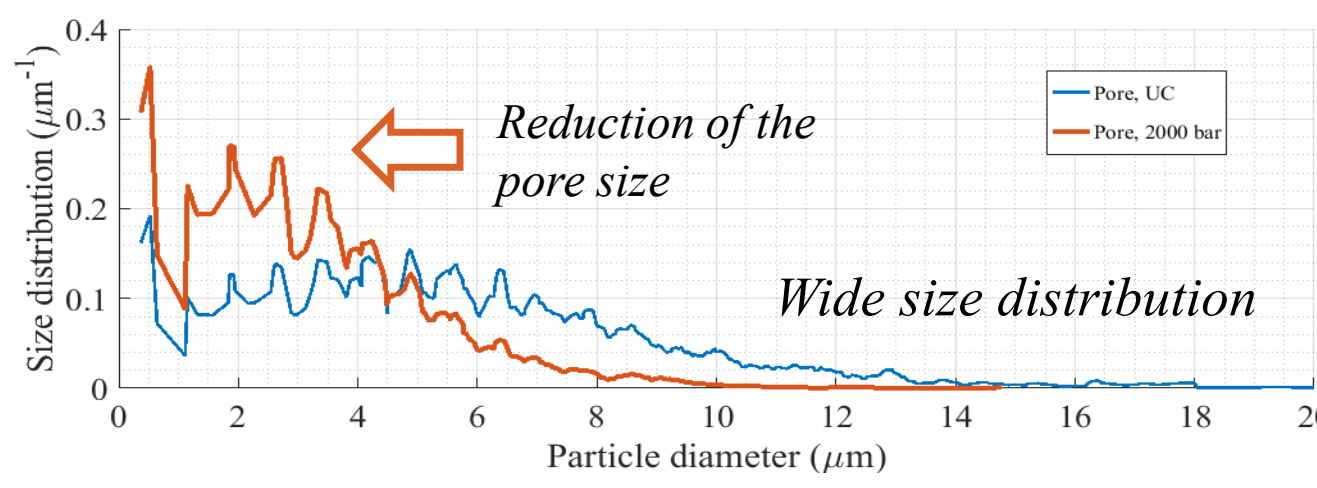




\section{Particle size: Representative volume element (RVE) size}

Correlating RVE size of $d_{50}$ with particle size: $d_{50} \& \operatorname{std}(d)$
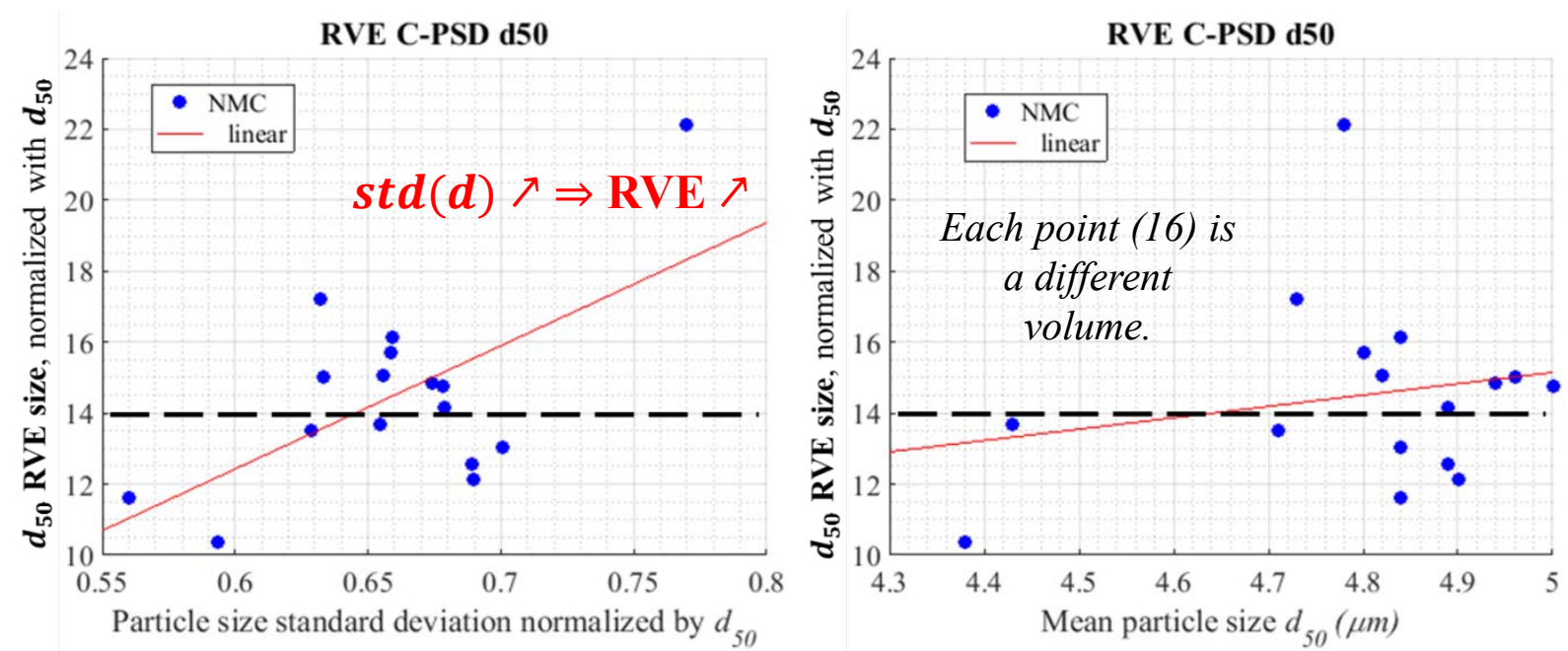

NMC (RVE

$\left.D_{50}\right)$ normalized

with $d_{50}$

Each point (16) is

a different

volume. 


\section{Particle size: Representative volume element (RVE) size}

\section{Correlating RVE size of $d_{50}$ with particle size: $d_{50} \& \operatorname{std}(d)$}
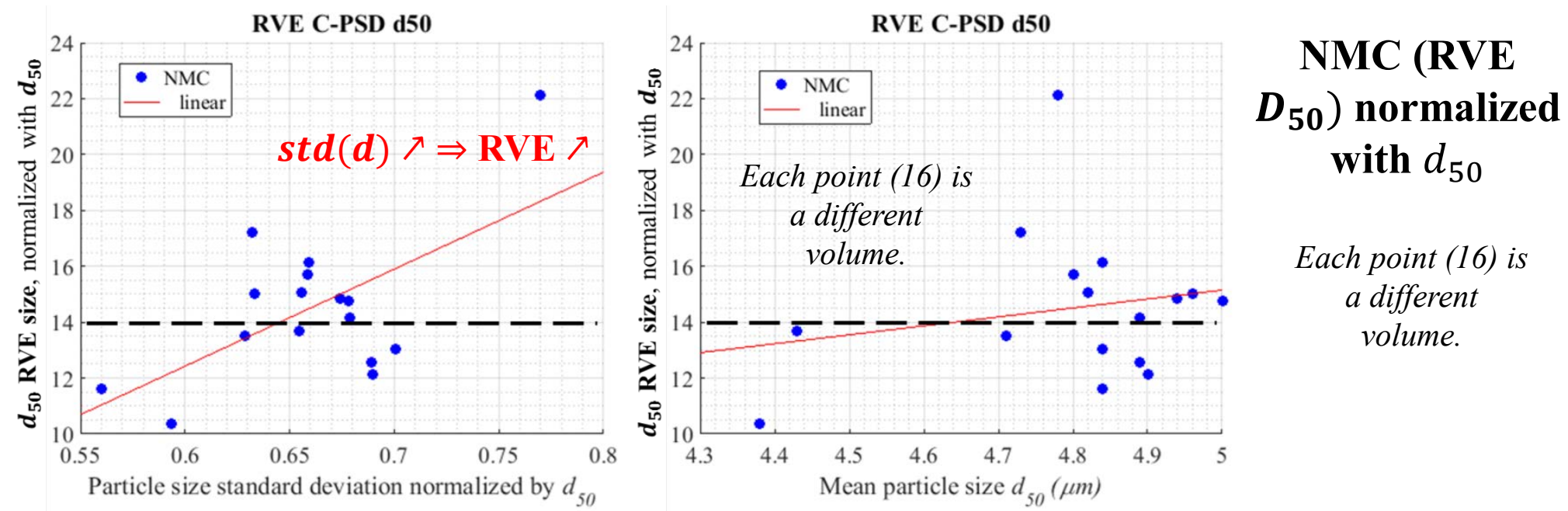

NMC (RVE $\left.D_{50}\right)$ normalized with $d_{50}$

Each point (16) is a different volume.

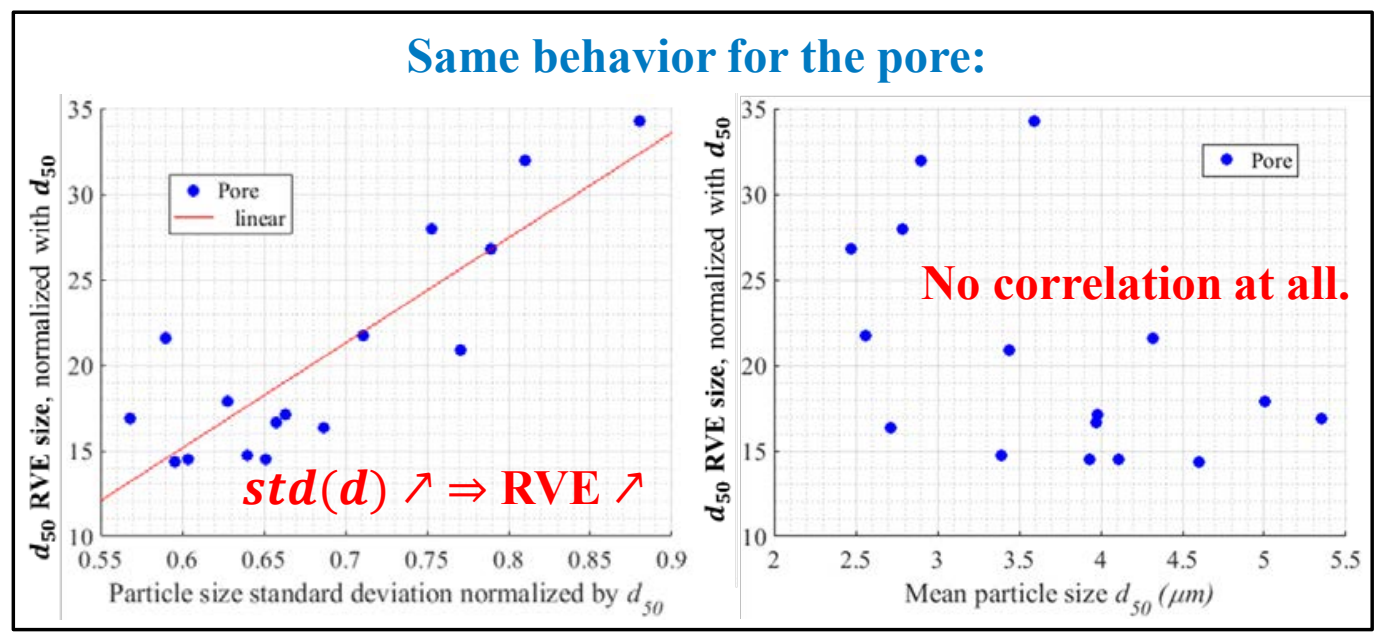

RVE, by definition, must catch all heterogeneities of the medium $\rightarrow$ the more heterogeneous the medium is, the larger the RVE will be.

Simple rule $\mathrm{RVE}=14 \times \boldsymbol{d}_{50}$ * is not enough for medium with a wide size distribution 


\section{Particle size: statistical approach \& watershed method}

\section{Alternative methods to avoid particle size underestimation}

Statistical approach

- Based upon a two-point correlation (covariance) function.

- Spherical assumption is no more used. But less detail are available (no size distribution)

- Refinement added to the classic method to improve the determination of the mean particle and pore size.

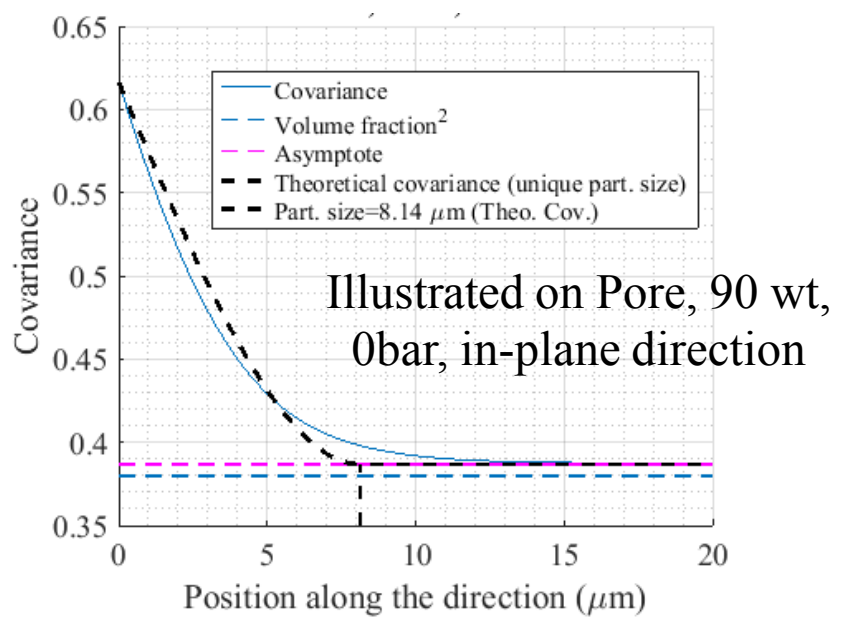

Detailed in the back-up slides to save presentation time, and in the upcoming ECTS article 


\section{Particle size: statistical approach \& watershed method}

\section{Alternative methods to avoid particle size underestimation}

Statistical approach

- Based upon a two-point correlation (covariance) function.

- Spherical assumption is no more used. But less detail are available (no size distribution)

- Refinement added to the classic method to improve the determination of the mean particle and pore size.

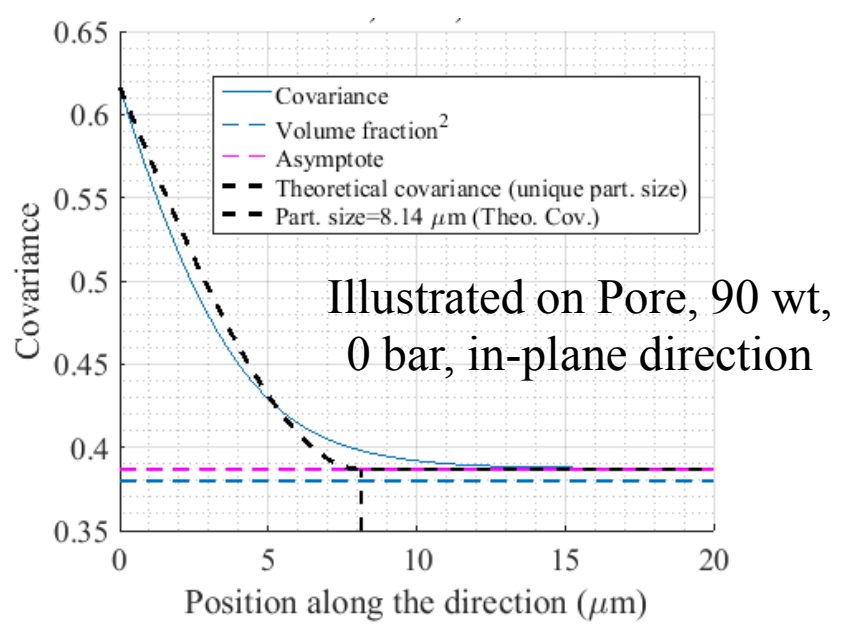

Watershed method

- Based upon an immersion approach, with a refinement to handle over-segmentation

- Distinct particles are identified without any shape assumption.

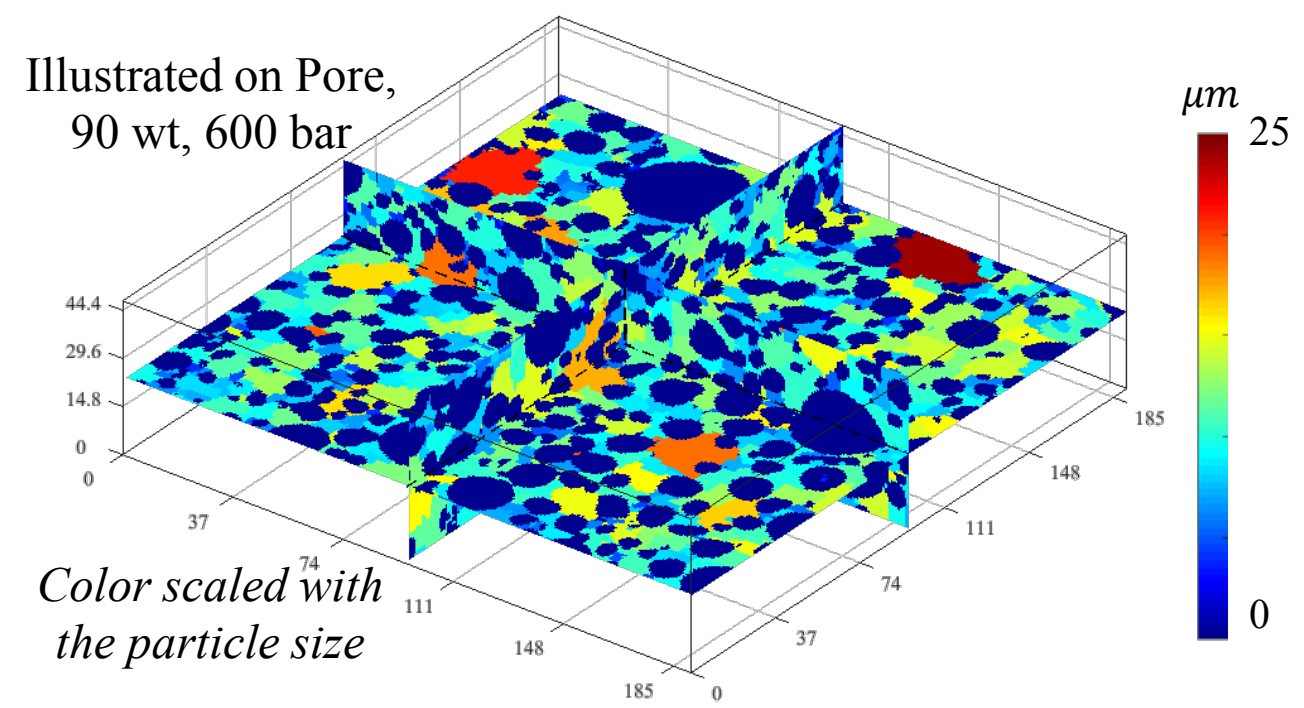

Detailed in the back-up slides to save presentation time, and in the upcoming ECTS article 


\section{Particle size: all three methods compared}

Particle size difference illustrated on the 90 wt electrode with different compression level
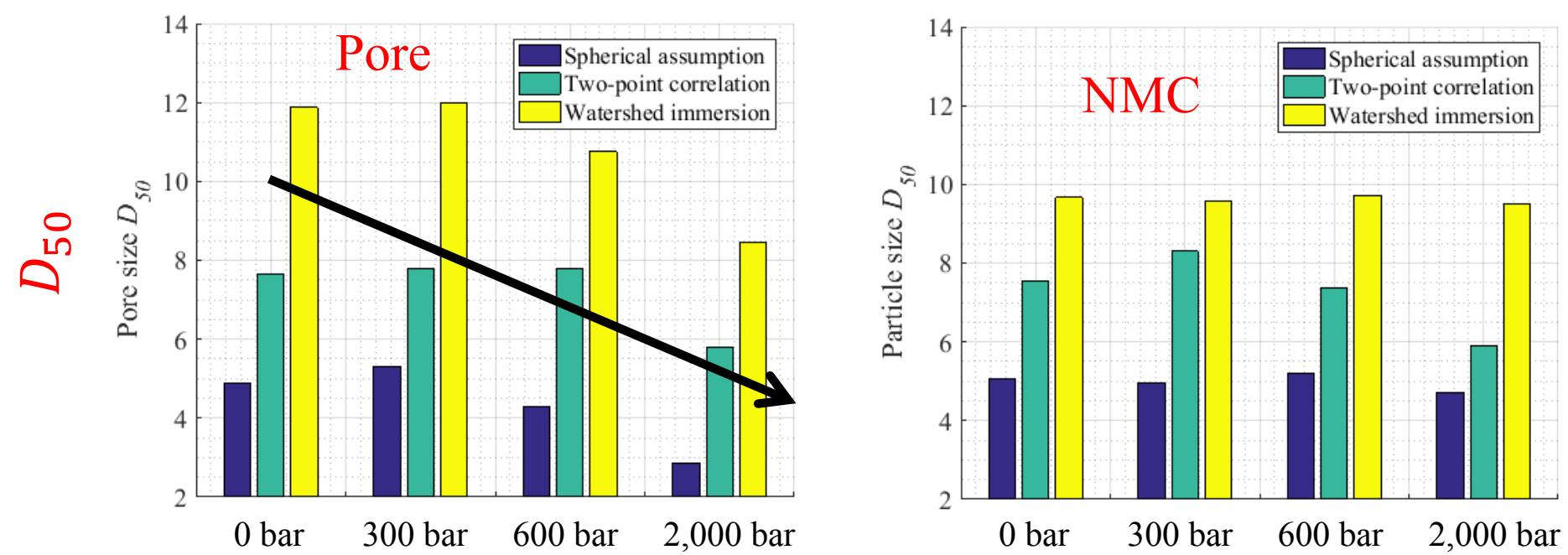

All methods
describe $D_{50} \searrow$ for
the pore with
compression

C-PSD
$<$
$<$
statistical approach
$<$
watershed




\section{Particle size: all three methods compared}

\section{Particle size difference illustrated on the 90 wt electrode with different compression level}
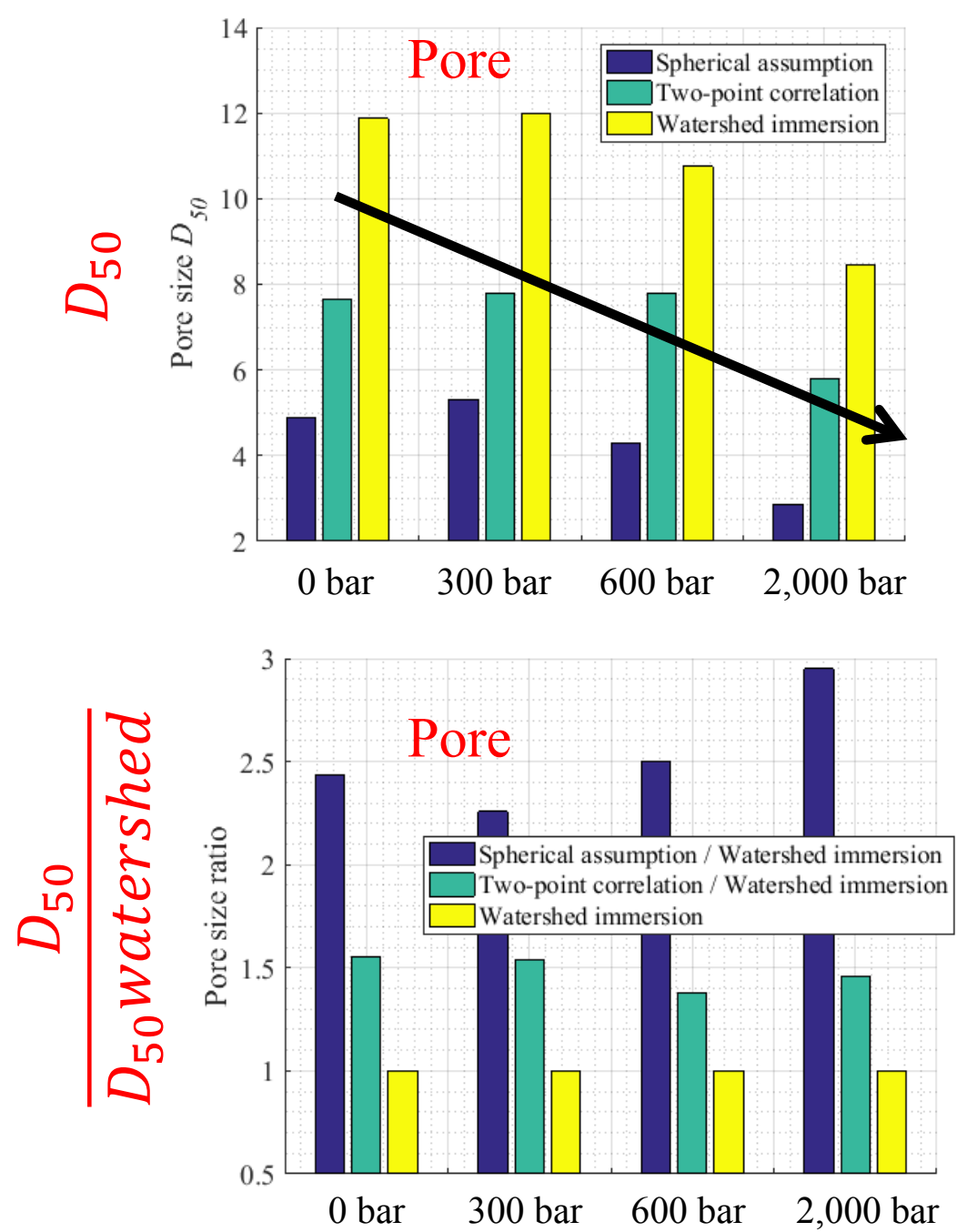
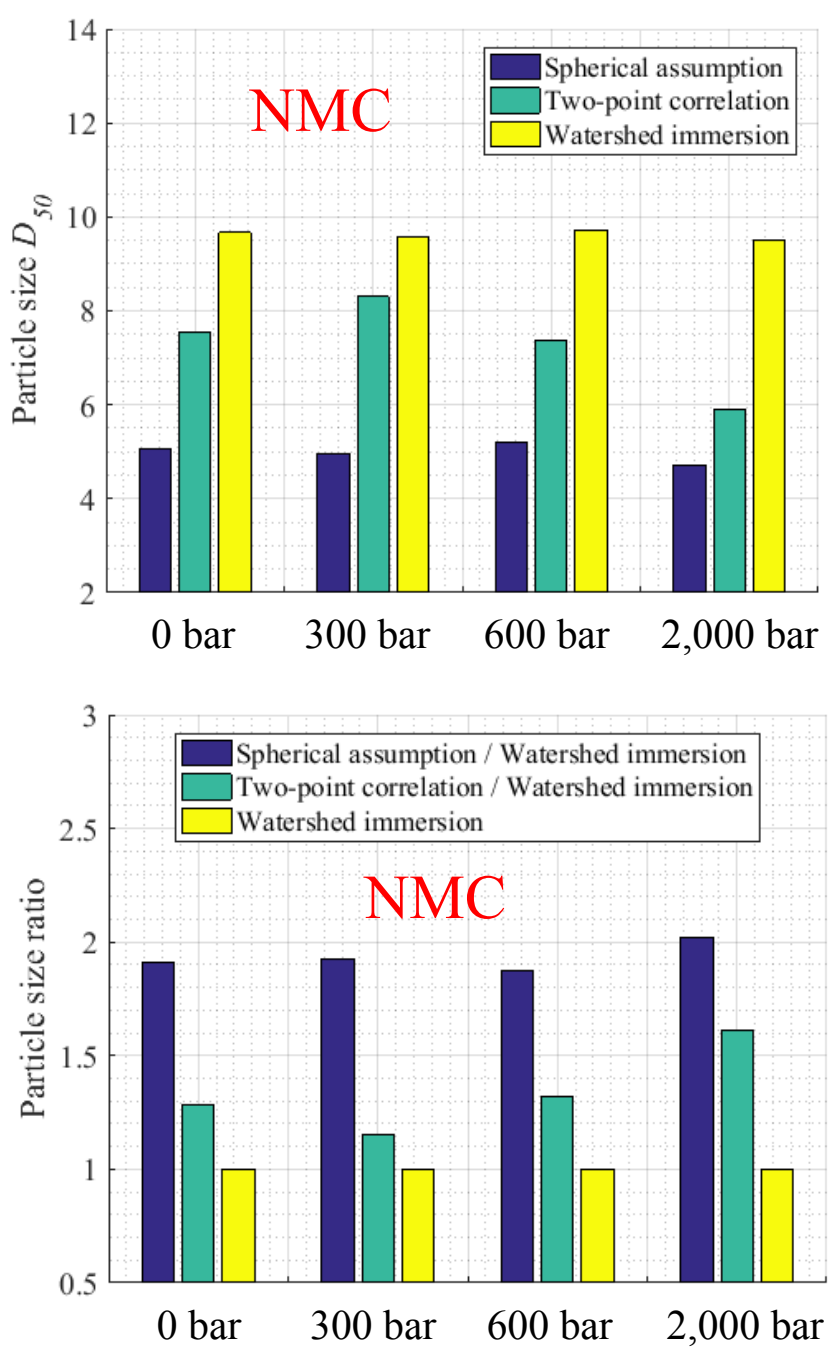

All methods describe $D_{50} \searrow$ for the pore with compression

\section{C-PSD $<$ \\ statistical approach $<$ watershed}

Ratio is quite high, but expected $\sim 2.8$ found be L. Holzer et al. JPS 196 (2011) 7076-7089

Ratio is higher for Pore than for NMC $\rightarrow$ Pore are less spherical than NMC particles. NMC are not so spherical. 


\section{Factor of tortuosity: voxel size dependence analysis}

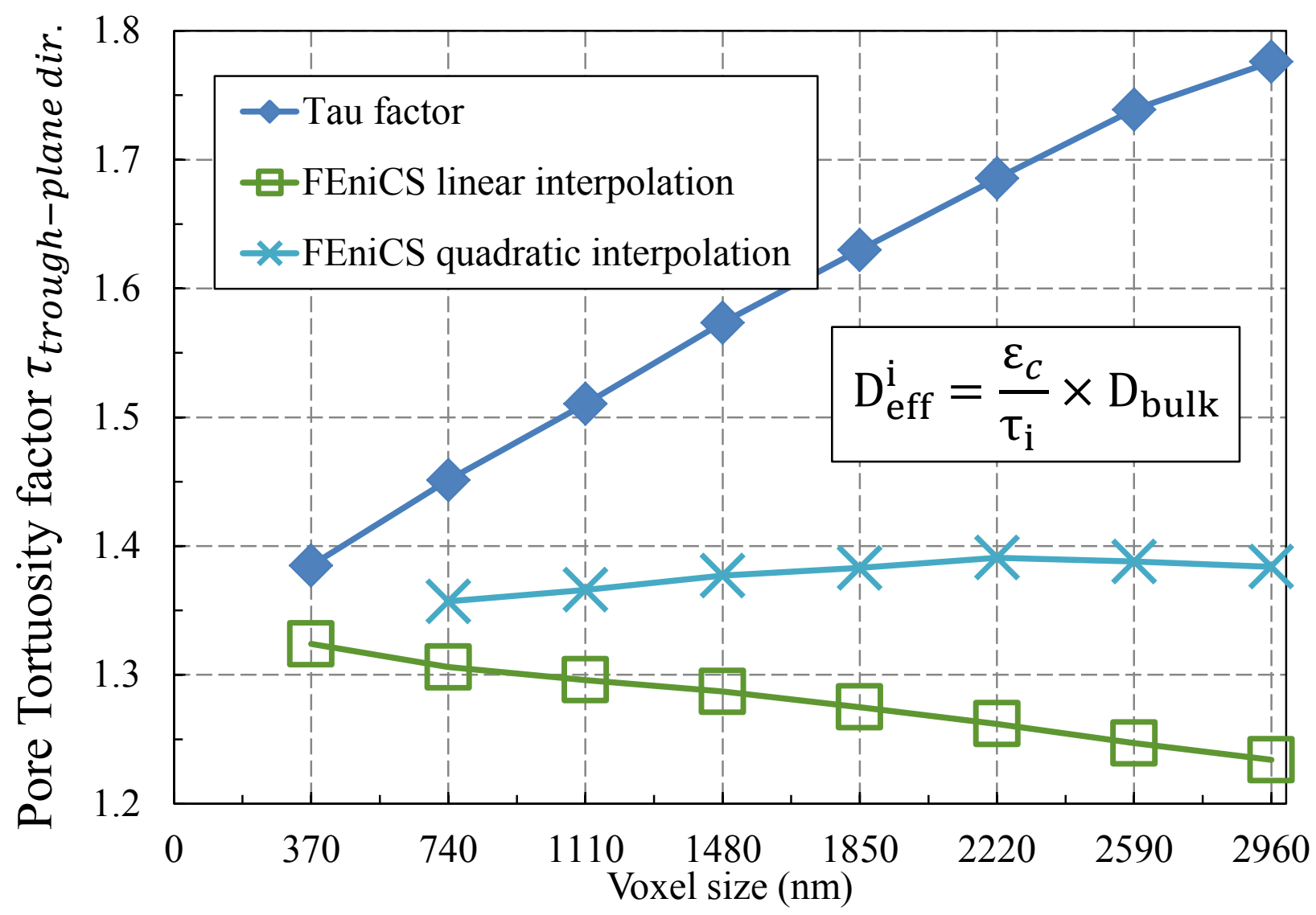

- Both methods (FEM \& neighbors difference) are converging to the same value.

- FEniCS has been used since it allows applying different boundary conditions.

- Save CPU time and memory: FEniCS with a linear interpolation and a voxel size of $740 \mathrm{~nm}$.
Tau factor (open source) iteratively solves the Laplace equation, using a neighbors difference, with Dirichlet Boundary conditions and a great emphasis on speed convergence S.J. Cooper et al., Software X 5 (2016) 203-210

FEniCS (open source) solves steady-state Laplace equation with the finite elements method. Dirichlet BC used in this figure.

Voxel size analyze performed on a pore subdomain $(\sim 185 \times 185 \times$ $\left.50 \mu^{3}\right)$ of the $90 \mathrm{wt}$ un-calendared electrode. 


\section{Factor of tortuosity: field of view and resolution achieved}

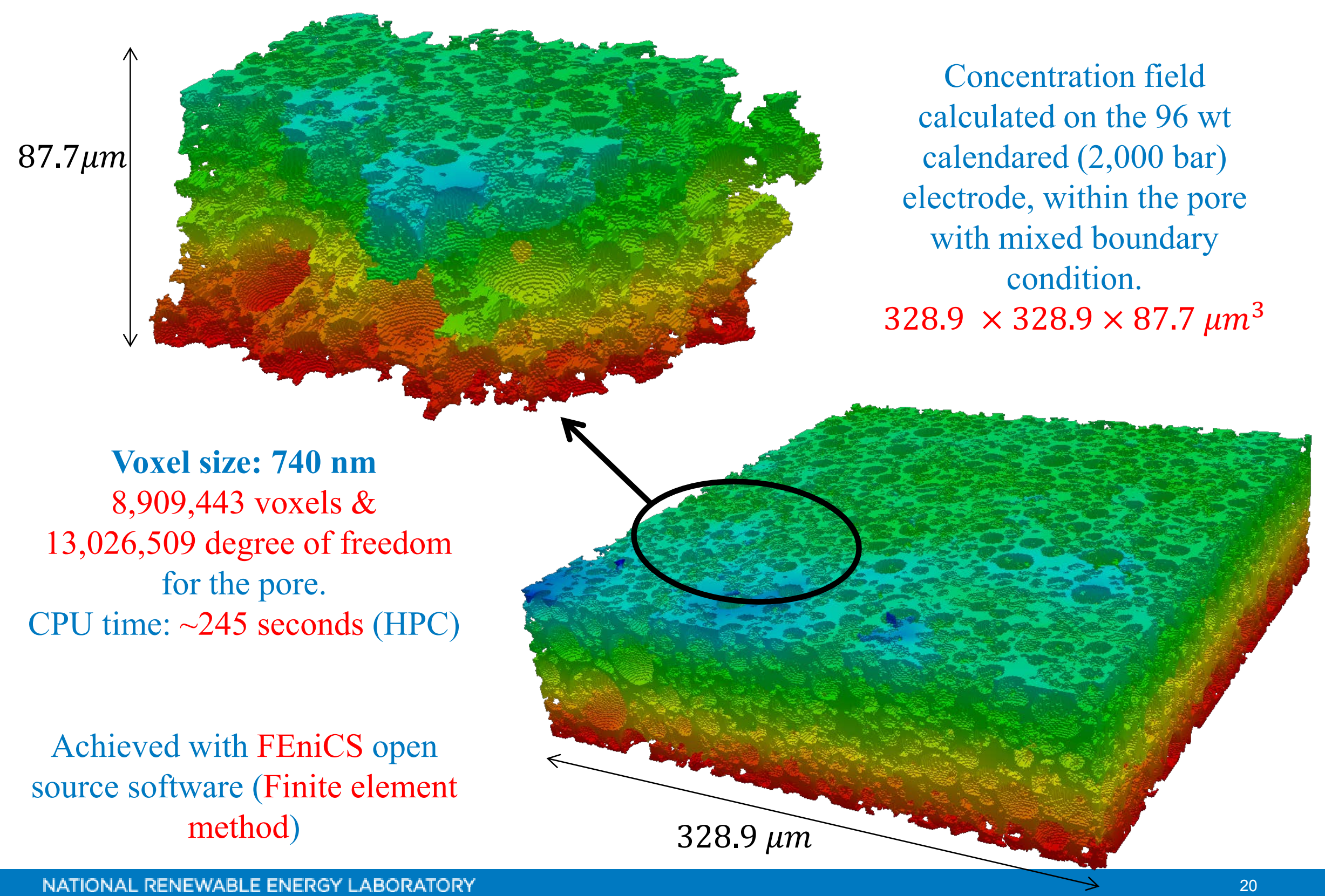




\section{Factor of tortuosity: Representative volume element analysis}

- RVE analysis on $\boldsymbol{\tau}_{\text {through-plane dir. }}$ with mixed BC (i.e. Dirichlet-Neumann)

Illustrated on $90 \mathrm{wt}, \mathrm{UC}$ and C (2,000 bar) for both phases
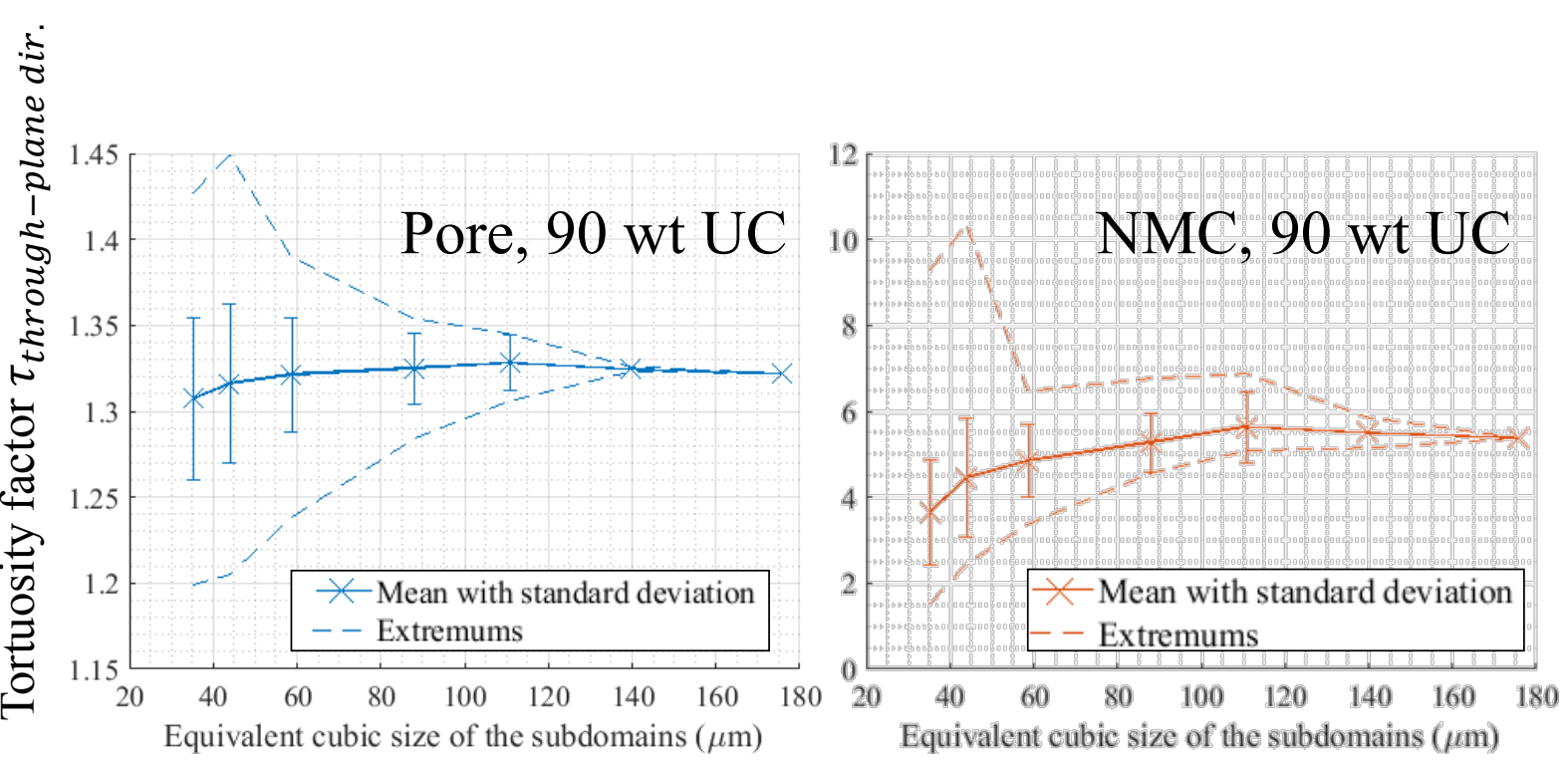

Tortuosity table (the RVE are written in parentheses)

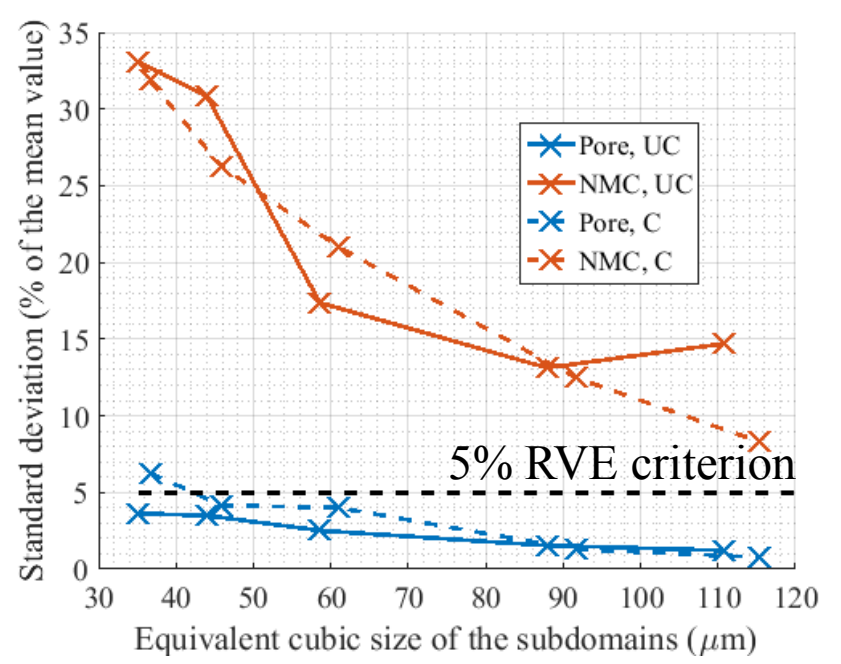

For which domain's size

$\tau_{\text {through-plane dir. }}$ is stable?

Equivalent cubic size of the subdomains $(\mu \mathrm{m})$ 


\section{Factor of tortuosity: Representative volume element analysis}

\section{- RVE analysis on $\boldsymbol{\tau}$ anisotropy with mixed BC}

Illustrated on $90 \mathrm{wt}, \mathrm{UC}$ and $\mathrm{C}$ (2,000 bar) for both phases
For which domain's size $\max \left\{\operatorname{abs}\left(\tau_{i}-\tau_{j}\right)\right\}$ is stable?
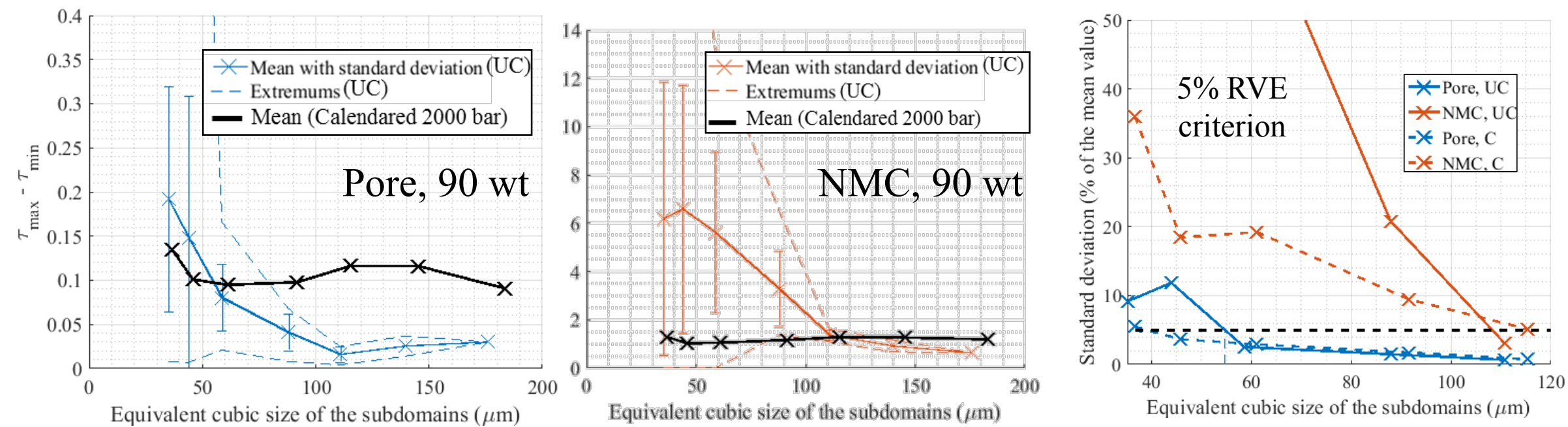

Isotropy is expected since solid phase is made of quasi-spherical spheres with a random space distribution.

For the un-calendared

Tortuosity anisotropy table (the RVE are written in parentheses)

\begin{tabular}{|c|c|c|c|c|c|c|}
\hline & \multicolumn{3}{|c|}{ Pore } & \multicolumn{3}{|c|}{ NMC } \\
\hline & & UC & C (2000 bar) & & $\mathrm{UC}$ & C (2000 bar) \\
\hline$\tau_{\max }-\tau_{\min }$ & 0.030 & $(54.7 \mu \mathrm{m})$ & $(39.0 \mu \mathrm{m})$ & 0.63 & $(108.3 \mu \mathrm{m})$ & $1.194 \quad(115.4 \mu \mathrm{m})$ \\
\hline
\end{tabular}

\section{RVE $\tau_{\text {anisotropy }} \approx \operatorname{RVE} \tau_{\text {through-plane dir }}$}

(While being inferior for the NMC) electrode, subdomains are anisotropic while whole domain is isotropic: isotropy emerges only for large volume

Calendaring affects isotropy

RVE on boundary conditions has been also evaluated (back-up slides) 


\section{Factor of tortuosity: rule of mixture $\&$ percolation loss}

\section{Why factor of tortuosity obtained on subdomains differs from the whole domain?}

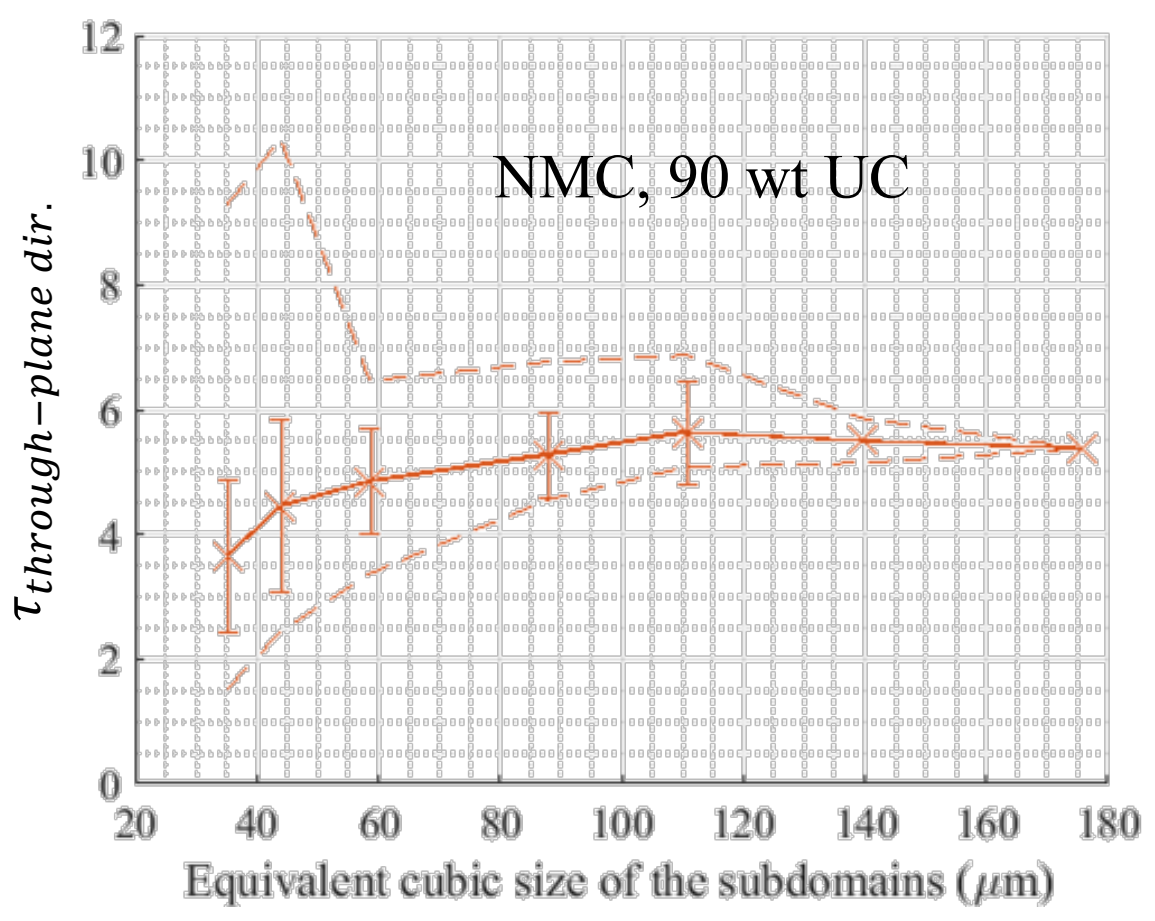

Loss of percolation on small subdomains should increase tortuosity by creating local bottleneck at the edges...but we see the opposite.

That's because taking the mean value (thus performed a rule of mixture) is a poor idea...it is true only when the subdomains are assembled in parallel with the flux (details in the annexes slides) 


\section{Factor of tortuosity: two-step homogenization numerical scheme}
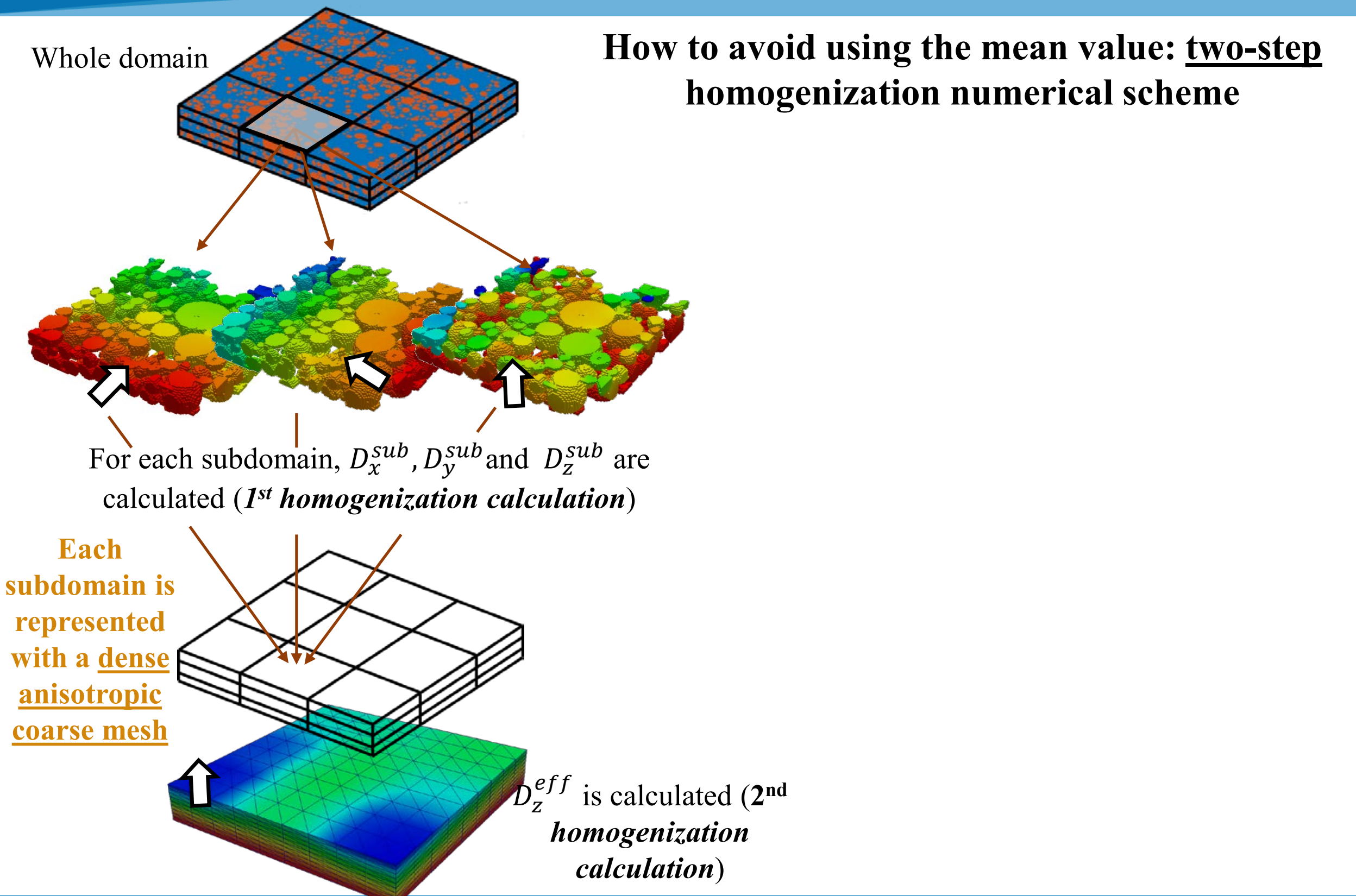


\section{Factor of tortuosity: two-step homogenization numerical scheme}

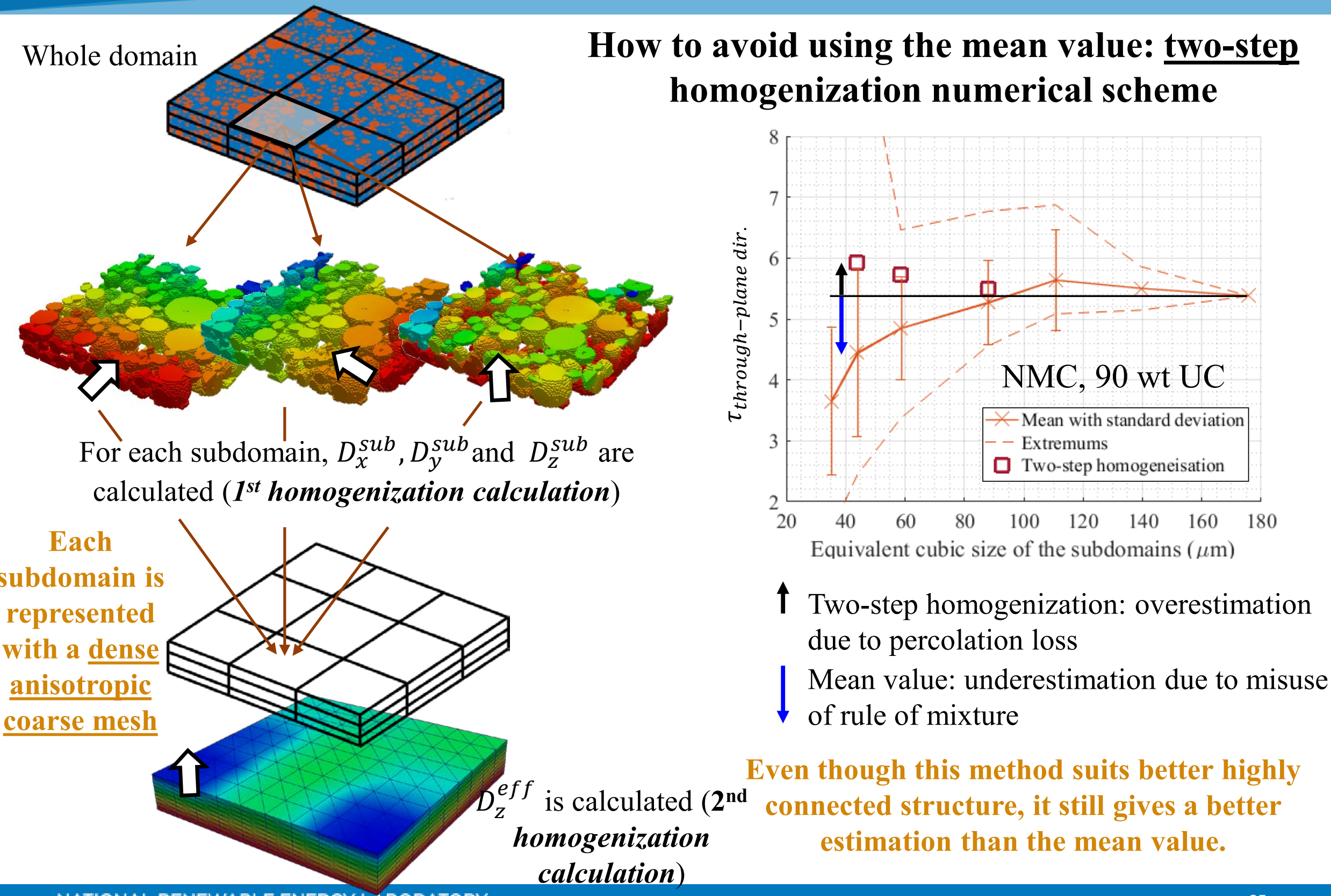




\section{Factor of tortuosity: calendaring effect}

0

Bar

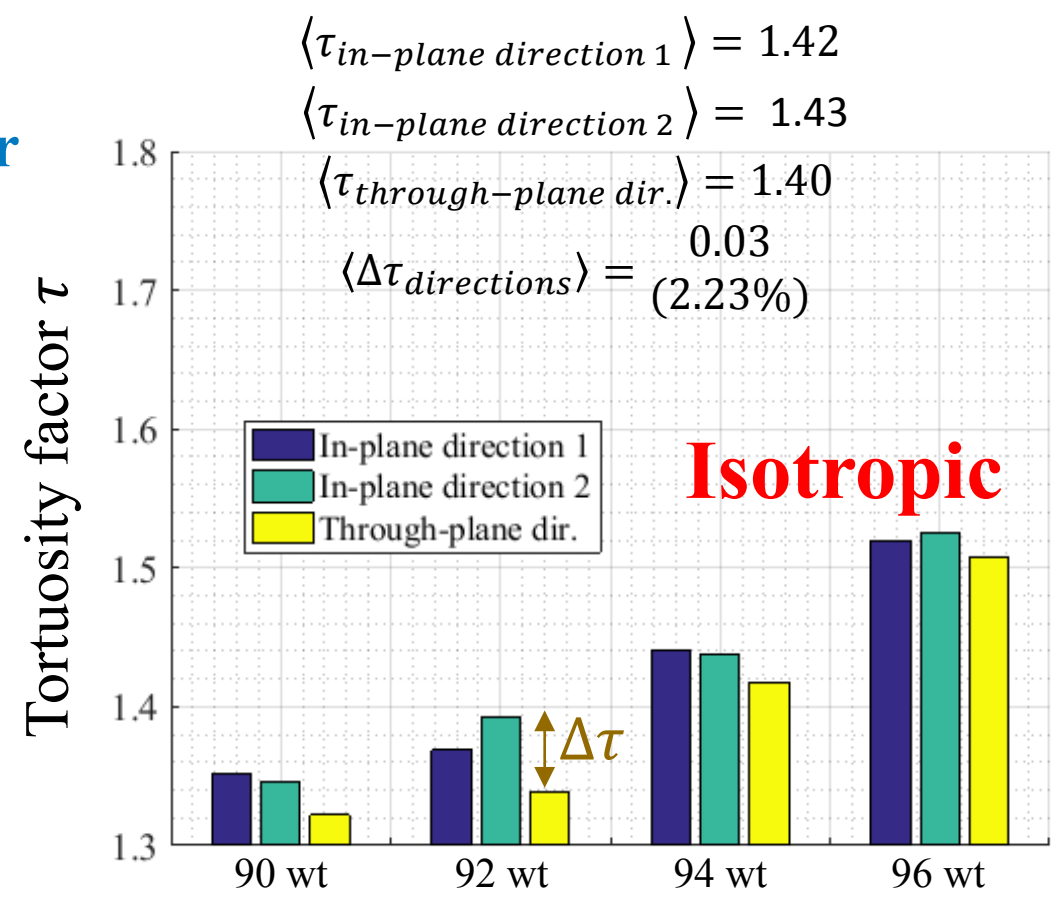

Calendaring induce a general increase of the tortuosity, due to the decrease of the connected porosity (Bruggeman exponent $\mathbf{0 . 6 5}$ ) \&

a slight anisotropy between in-plane directions and through-plane direction

(could be induced by a change of NMC particle shape)
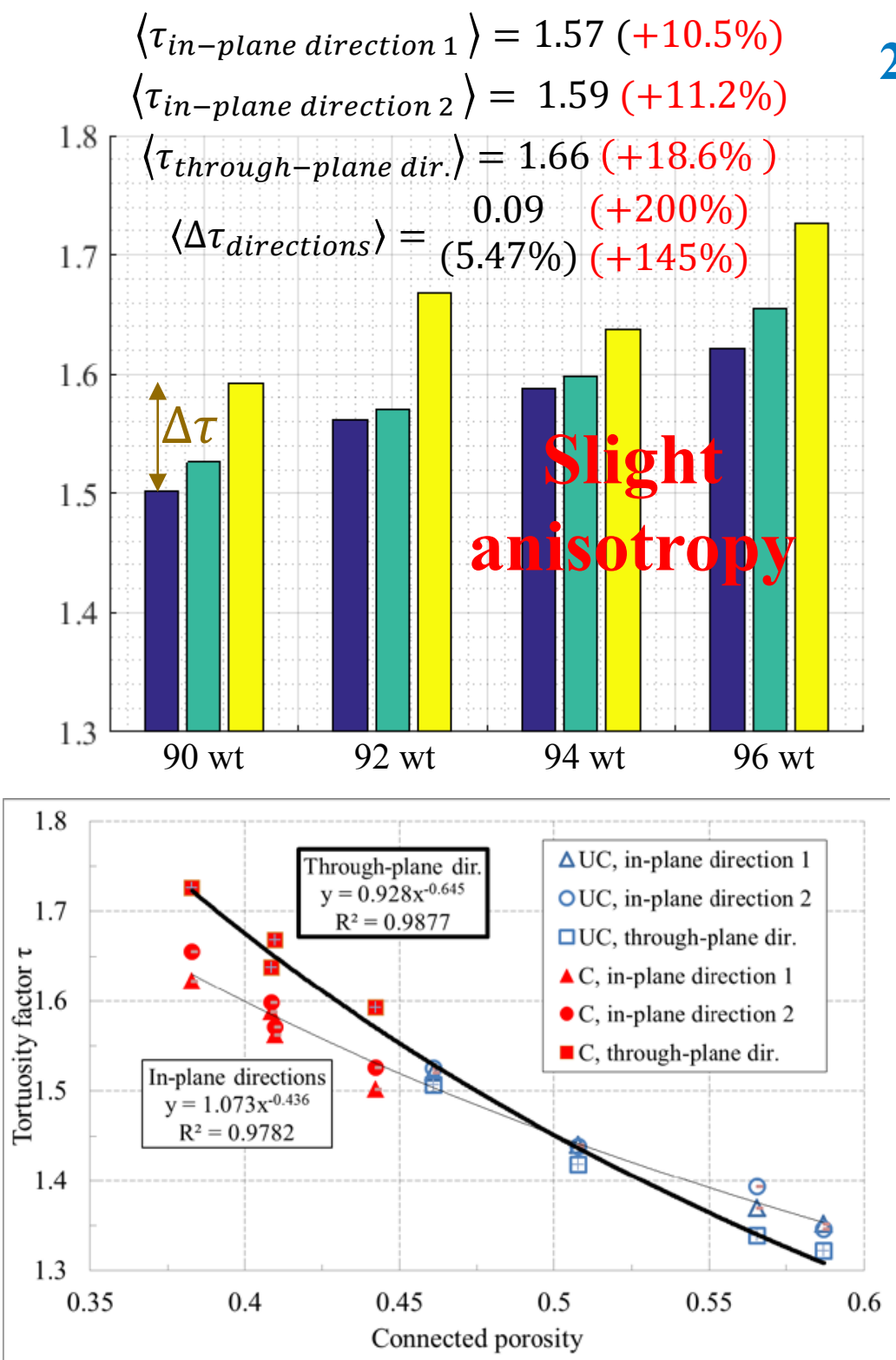


\section{Conclusions 1/2: numerical aspects}

- RVE

$>$ Are property-dependent

$>$ Simple rule such as RVE size $=\mathrm{x}$ times mean particle size doesn't stand for structure with wide size distribution. RVE size increases with heterogeneity (i.e. standard deviation of parameters)

- Particle size and surface area (annexes)

$>$ Dependent on the voxel size.

$>$ The product $S_{p} \times d_{50}$ is less sensitive to the voxel size and exhibits more similar values compared with $S_{p}$ and $d_{50}$ taken individually: it is thus a better identifier of the microstructure.

- Bias \& assumption

$>$ Connectivity suffers from a border effect. It should be monitored on small domains.

$>$ Particle size depends a lot on the chosen method. One should not rely on a unique approach.

$>$ Spherical assumption is incorrect both for Pore and NMC. Shown for particle size: pore are less spherical than NMC, which are themselves not really spherical. Results obtained on the product $S_{p} \times d_{50}$ goes to the same direction (annexes) 


\section{Conclusions 2/2: practical information}

- Microstructure observation

$>$ Image quality of the segmented data set can be evaluated with the image level of detail parameters.

$>$ Consider standard deviation of the particle size when choosing your field of view.

- Calendaring pressure

$>$ Increases solid phase connectivity

$>$ Reduces the pore size

$>$ General increase of the tortuosity + slight anisotropy that hinder the diffusion in the through-plane direction (likely due to particle rearrangement and/or change of particle shape), something we would prefer to avoid.

- Modeling

$>$ 1D-model could take advantage of spatially varying parameter along the electrode thickness (as illustrated for tortuosity).

- Tortuosity determination for large domain

$>$ Consider using the two-step homogenization method to analyze if your domain is too big (or if you don't have access to an HPC). It's better than relying on a rule of mixture. 


\section{Thank you for your attention}

Any questions? 


\section{Annexes: RVE}




\section{Determination of the RVE based upon subdomains}

\section{Representative volume element analysis illustrated on volume fractions $\varepsilon$}

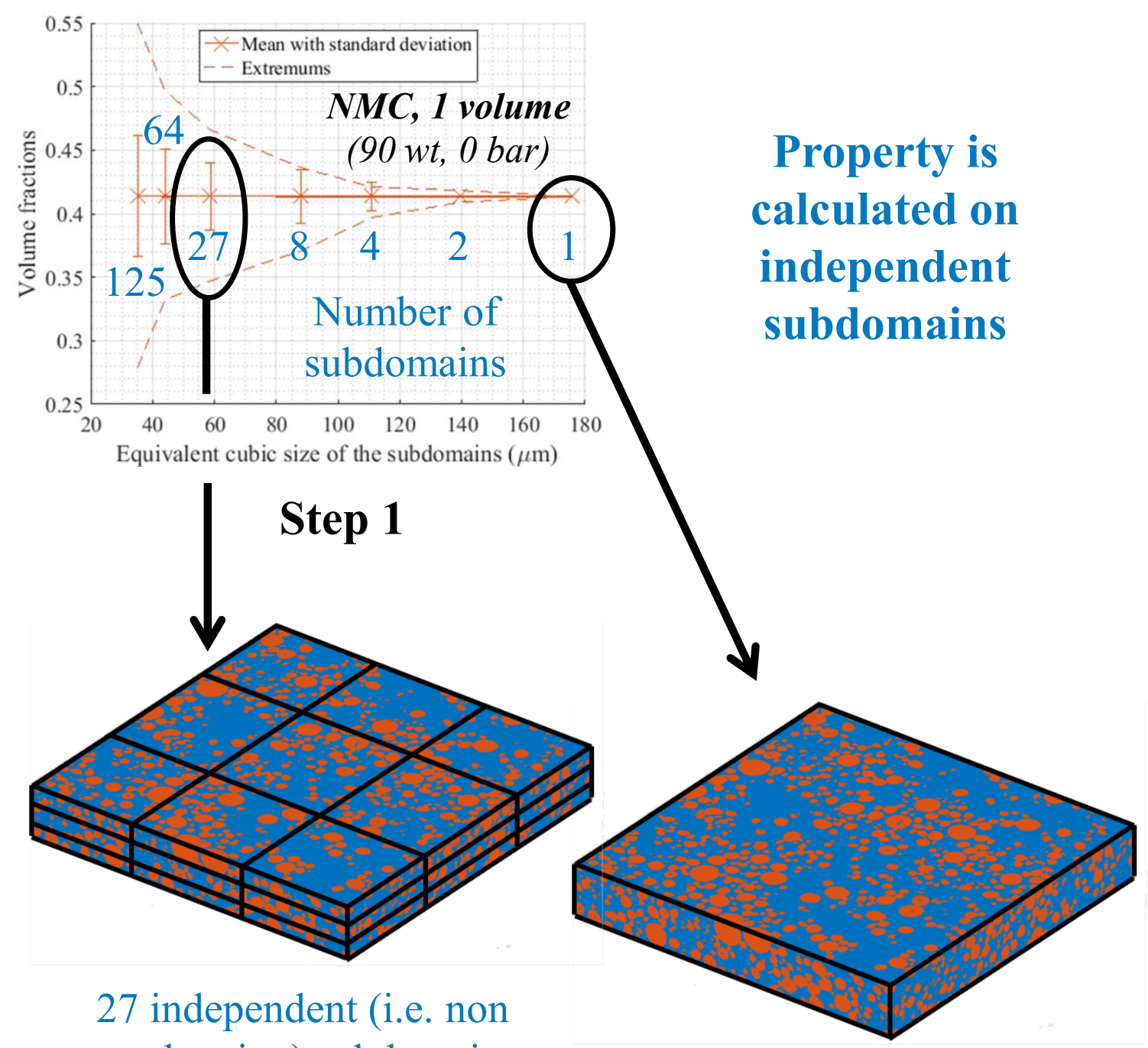

overlapping) subdomains

Whole domain 


\section{Determination of the RVE based upon subdomains}

\section{Representative volume element analysis illustrated on volume fractions $\varepsilon$}

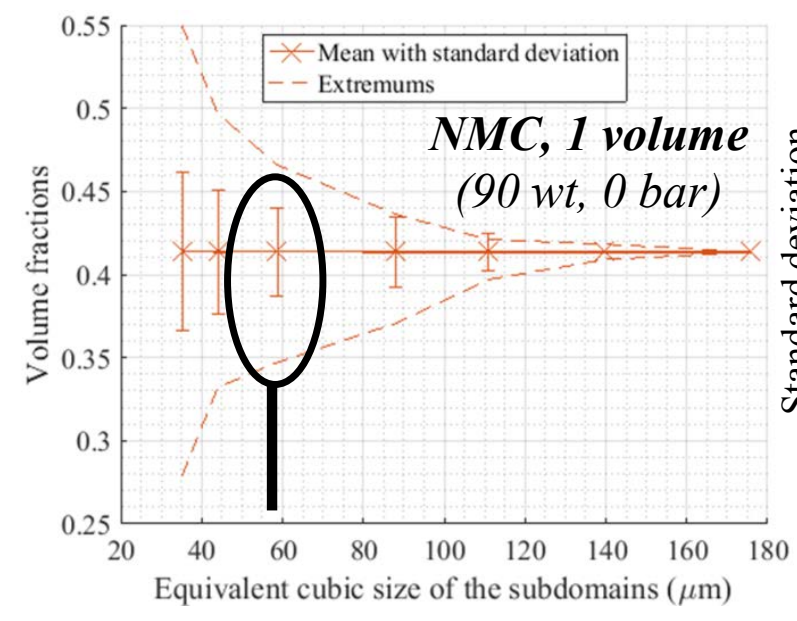

$\sqrt{2}$

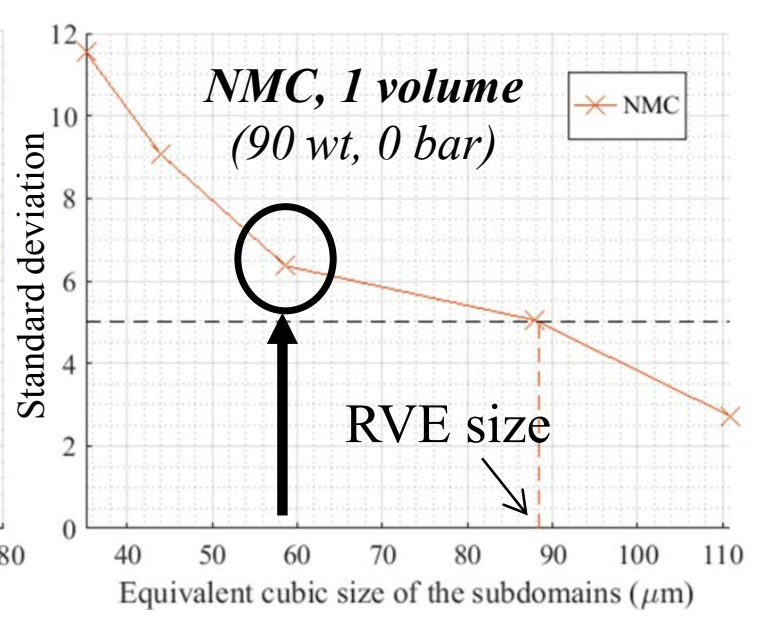

Step 2

Standard deviation obtained on subdomains is expressed in \% of the mean value

When the std is lower than a critical value (5\%)

then the volume is large enough to be representative: it is the RVE size 


\section{Determination of the RVE based upon subdomains}

\section{Representative volume element analysis illustrated on volume fractions $\varepsilon$}
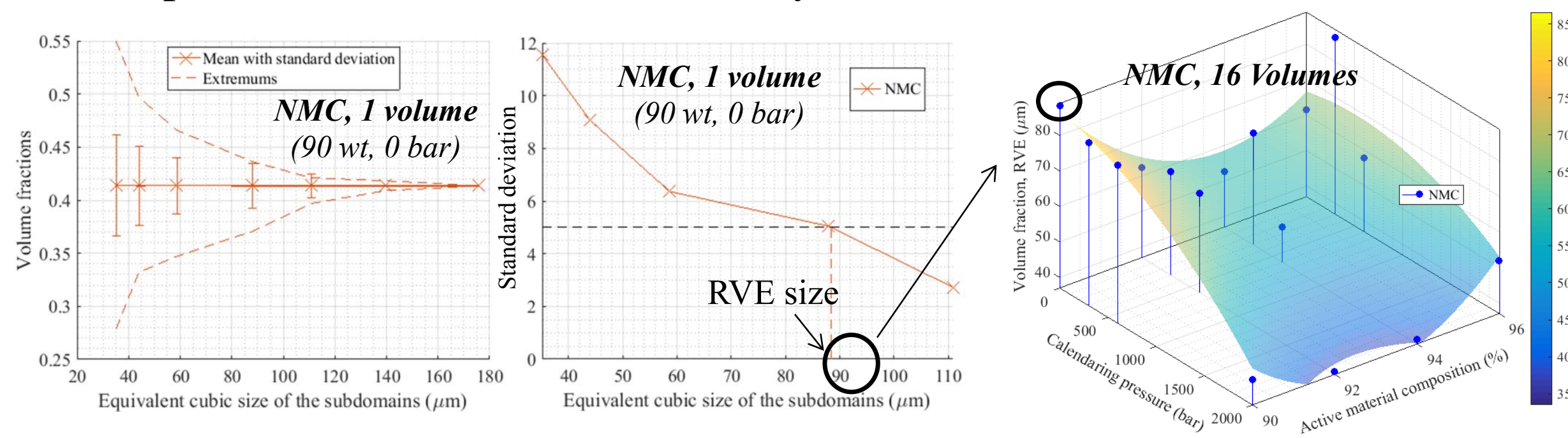

Step 1

Step 2

Step 3

Quadratic polynomial surface

The RVE size obtained on each microstructure (16 volumes) are plotted as a function of the calendaring pressure fit on RVE size and of the active material composition.

Similar analysis have been performed for the other properties $\sim 50 \mu \mathrm{m}<\mathrm{RVE} \varepsilon$ Pore $<\sim 100 \mu \mathrm{m}$ 


\section{Annexes: Surface area}




\section{Specific surface area}

- Based upon a direct counting method (overestimation corrected with a $2 / 3$ factor that use a spherical assumption)

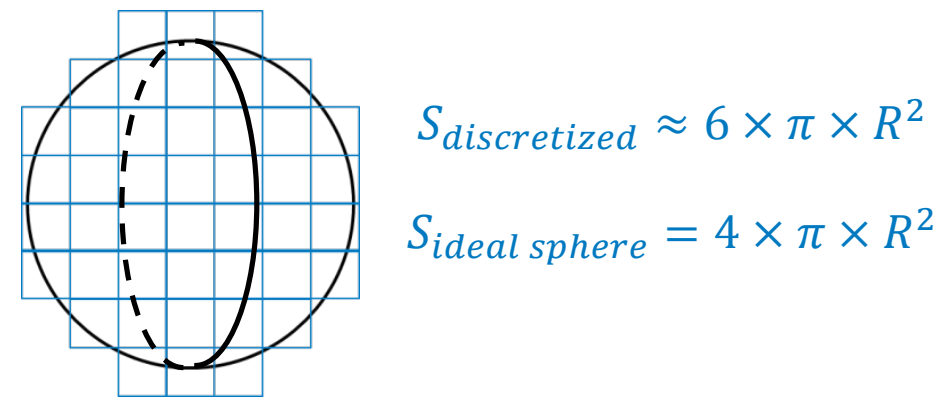

- Alternative method based upon the geometrical covariogram $K$ that gives the exact value without overestimation, but requires an isotropic assumption.

$$
K(V, \bar{h})=\operatorname{Mes}\left(V \cap V_{\bar{h}}\right)
$$

Surface area $=-4 \times\left.\frac{d(K(V, h)}{d h}\right|_{h=0}$

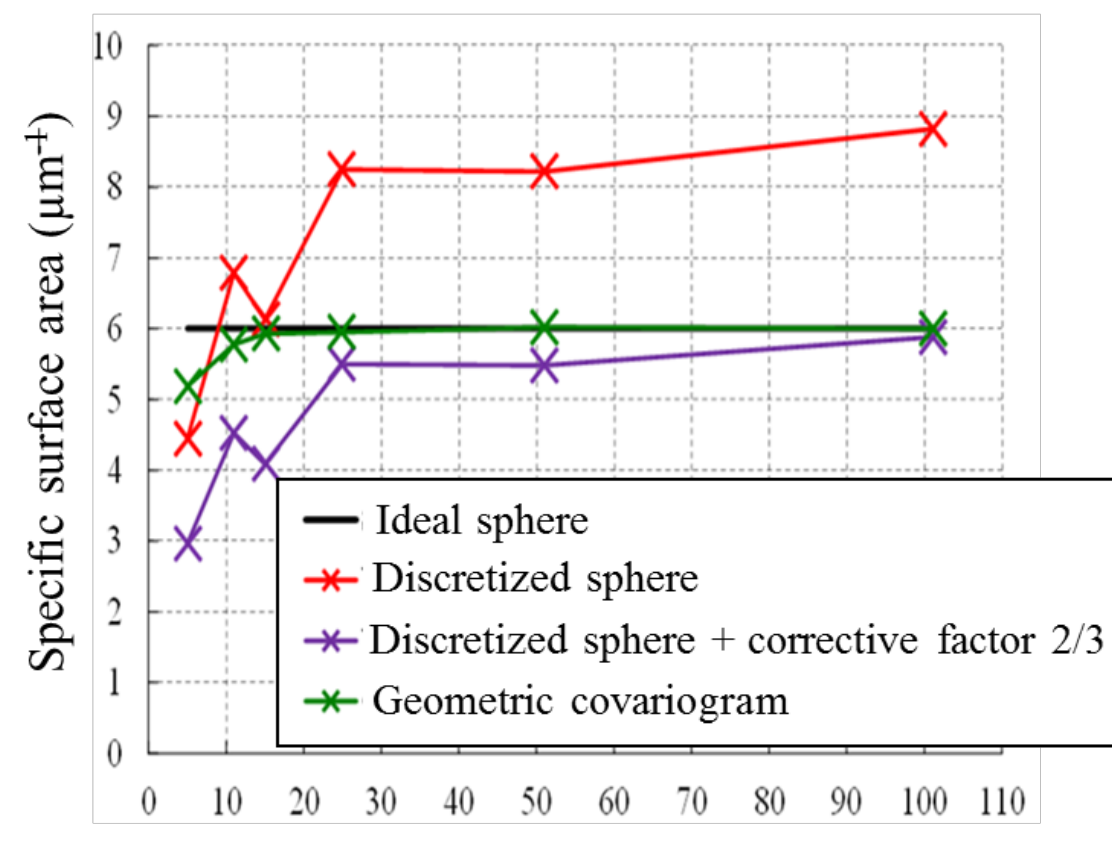

Number of voxel on the sphere diameter

Less voxel per sphere diameter are required to get the actual specific surface area with the geometric covariogram method.

A. Haas et al., Annales des mines XI, 736-753 (1967). 


\section{Specific surface area, geometric covariogram}

$K(V, \bar{h})=\operatorname{Mes}\left(V \cap V_{\bar{h}}\right)=\iiint_{V(h)} k(x, y, z) \times k\left(x+h_{1}, y+h_{2}, z+h_{3}\right) \times d V$ $A=-4 \times\left.\frac{d(K(V, h)}{d h}\right|_{h=0} \quad$ if $V$ isotropic with $\left\{\begin{array}{c}k(x, y, z)=\left\{\begin{array}{c}1 \text { if }(x, y, z) \in V \\ 0 \text { if }(x, y, z) \notin V\end{array}\right. \\ \bar{h}=h_{1} \overline{e_{x}}+h_{2} \overline{e_{y}}+h_{3} \overline{e_{z}}\end{array}\right.$
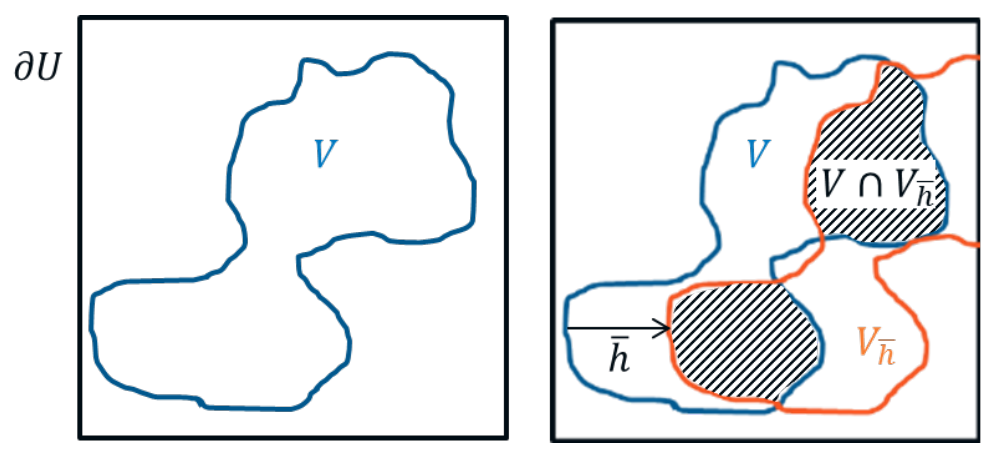

$$
S_{p}(V)=\frac{\delta V}{m e s(V)}=\frac{-4 \times\left.\frac{d K(V, h, \propto)}{d h}\right|_{h=0}}{\begin{array}{c}
m e s(V) \\
\text { if } V \text { isotropic }
\end{array}}
$$

No overestimation since the function is based upon a volume calculation. Volume does not depend on the medium discretization

A. Haas et al., Annales des mines XI, 736-753 (1967).

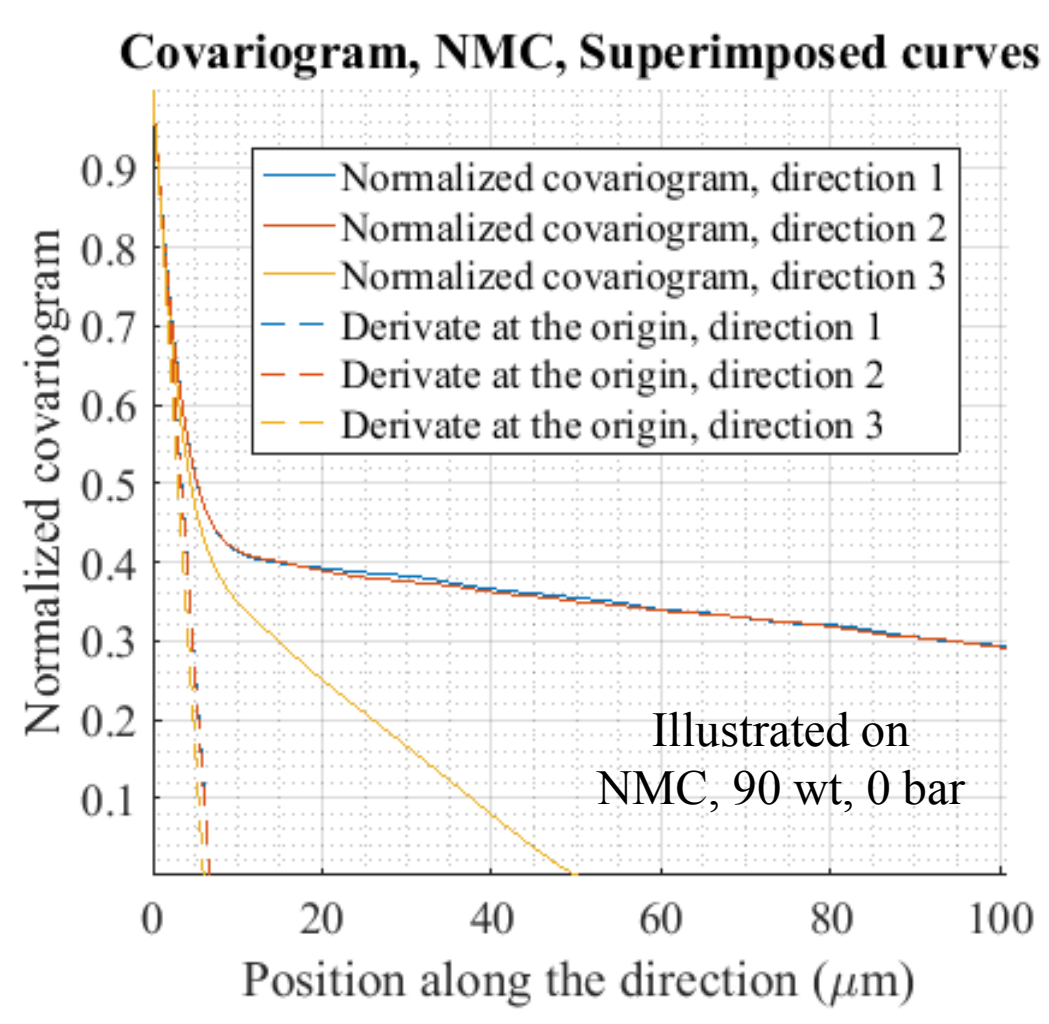

In the case the volume analyzed is a cube, this function can be used to evaluate the medium isotropy. 


\section{Specific surface area, results compared between methods}

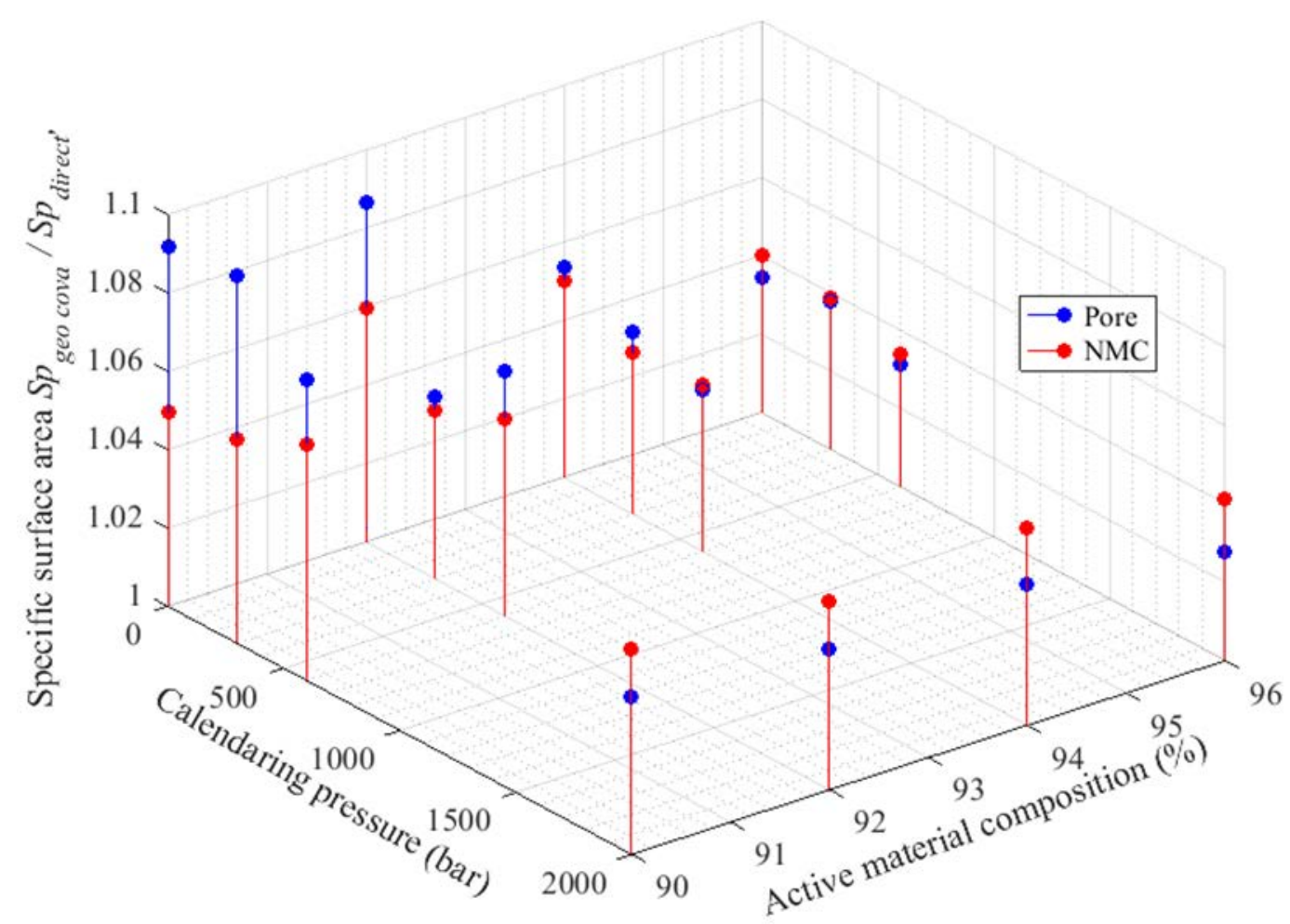

$1.028 \leq \frac{S_{p} \text { Geometric covariogram }}{S_{p} \text { direct method } \times 2 / 3} \leq 1.094$

Once the corrective factor $2 / 3$ is applied to the direct method, both methods provides very similar results.

$$
\text { (difference }<10 \% \text { ) }
$$
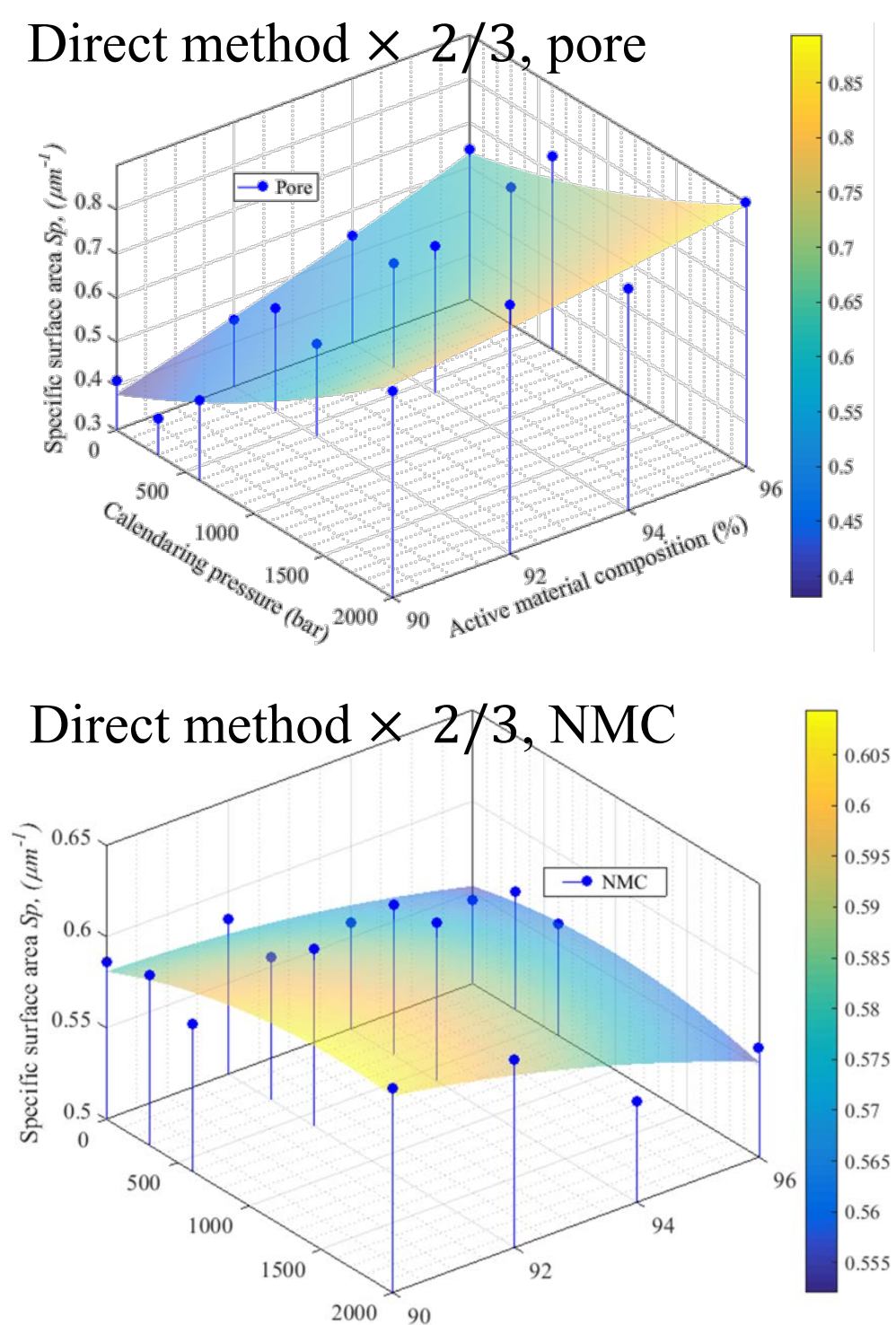

$S_{p}$ Pore $\nearrow$ with calendaring pressure. NMC stays quasi constant (from 0.55 to $0.61 \mu m^{-1}$ ) 


\section{Voxel size dependence illustrated on 90 wt 300 bar}
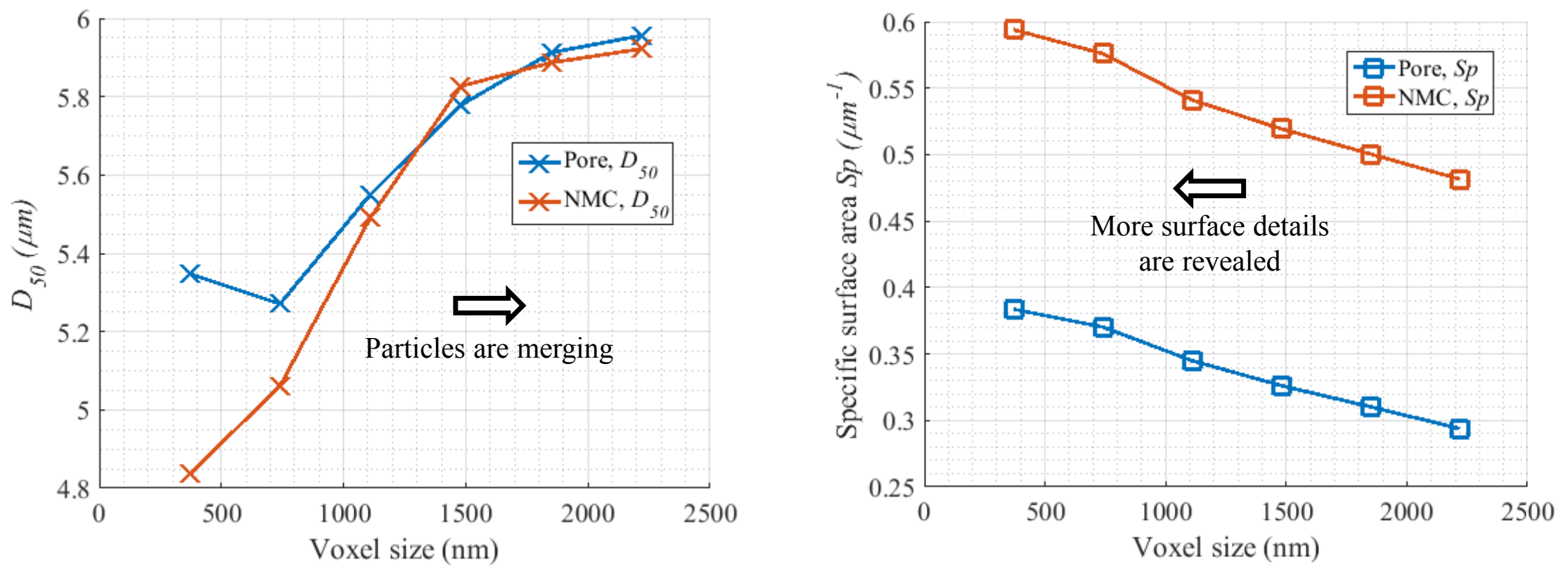

$>$ Both properties exhibit opposite voxel size dependence trend.

$>$ Ideally, particle $\&$ pore size will converge

but surface area will keep increasing (fractal property)

How to correlate them? 


\section{Particle size \& specific surface area correlation}

\section{Specific surface area as a function of $1 / d_{50}$}

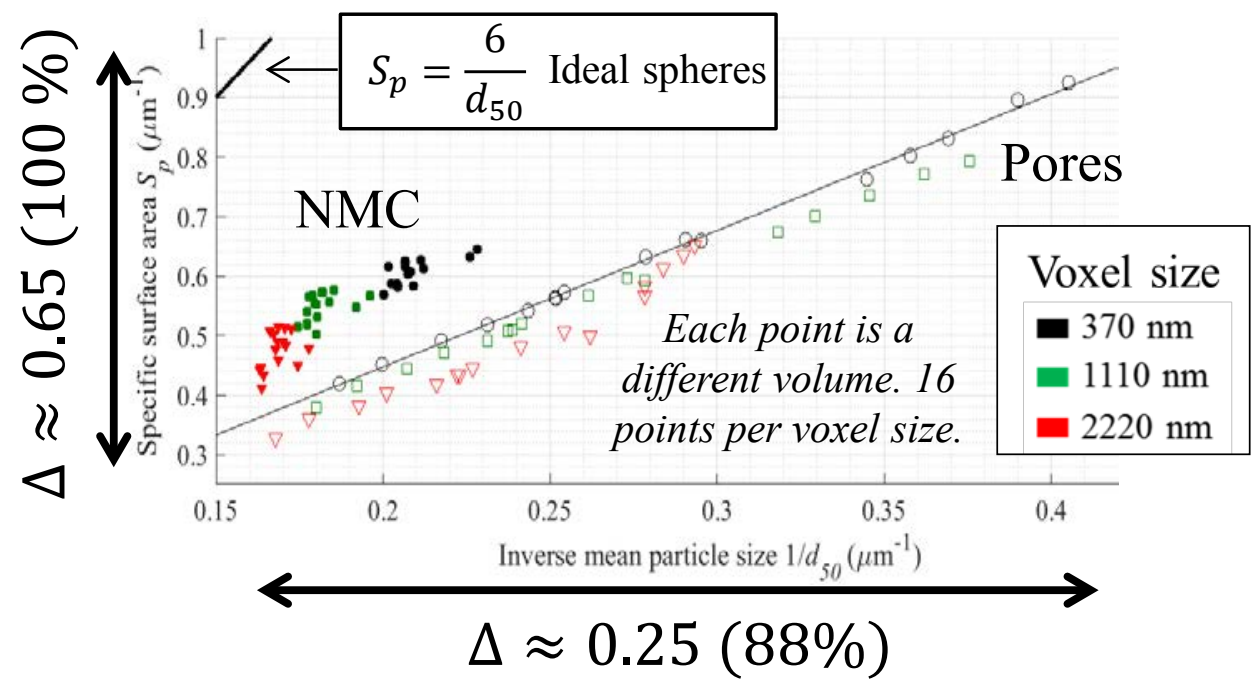

- The range of value for the pore is very large, both for $S_{p}$ and $d_{50}$ while it's basically the same microstructure.

- Linear correlation between $S_{p}$ and $1 / d_{50}$. Besides, the slope seems fairly constant with the image resolution.

$>$ Let's consider the slope $S_{p} \times d_{50}$ as a better identifier of the microstructure, instead of $S_{p}$ or $d_{50}$ alone.
Product $S_{p} \times d_{50}$

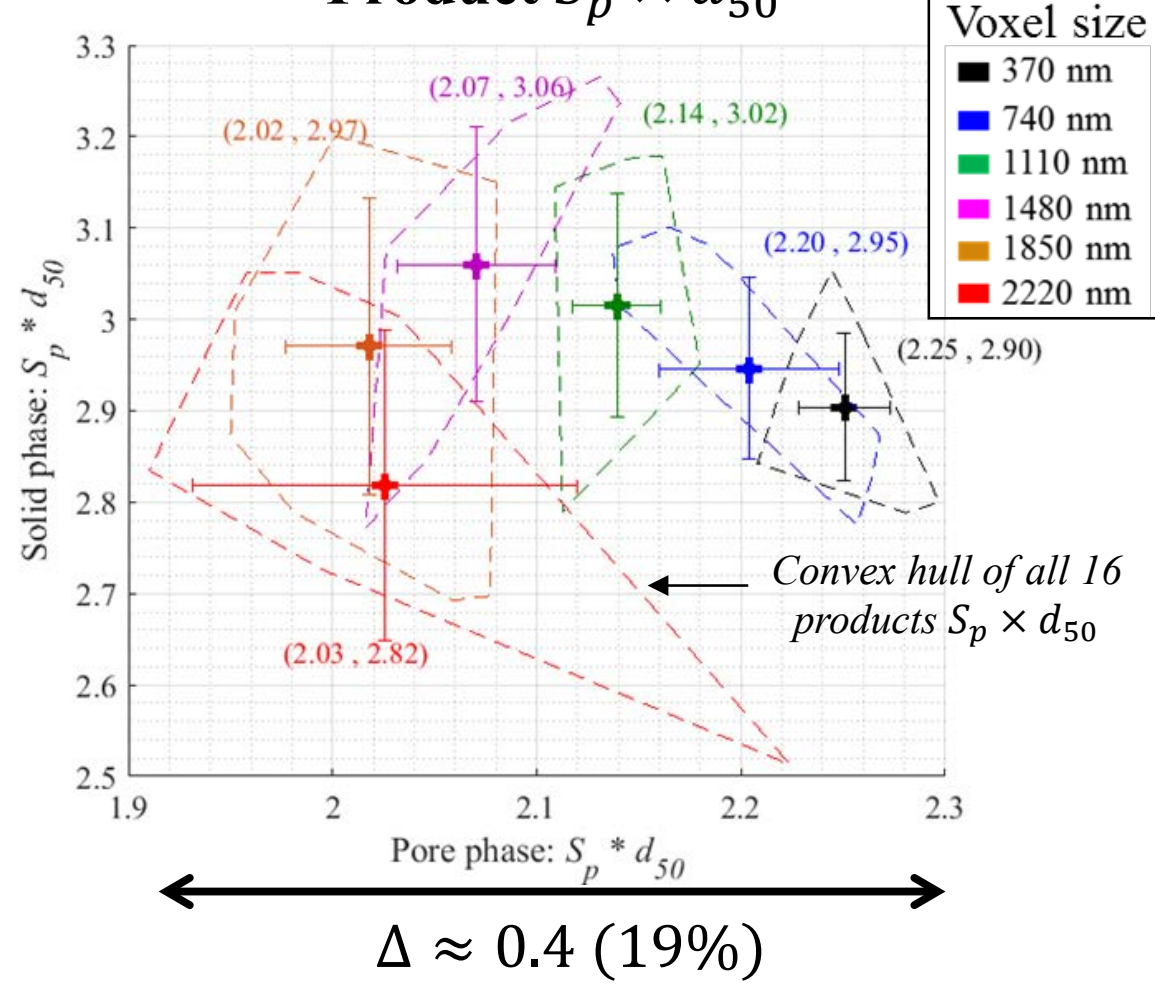

Product $S_{p} \times d_{50}$ voxel is less dependent with voxel size than $S_{p}$ or $d_{50}$ alone. Thus, the product better identifies a specific microstructure

$$
\begin{aligned}
& \left(S_{p} \times d_{50}\right) \quad\left(S_{p} \times d_{50}\right) \quad\left(S_{p} \times d_{50}\right) \\
& \text { pore }<\text { NMC }<\text { Spheres } \\
& 2.02-2.25 \quad 2.82-3.06 \quad 6
\end{aligned}
$$

Pore are less spherical than NMC particles. NMC are not so spherical. 


\section{Particle size \& specific surface area correlation}

\section{If they are intrinsically correlated, then they should share the same RVE size}

RVE size of all 16 volumes, for $S_{p}$ and $D_{50}$
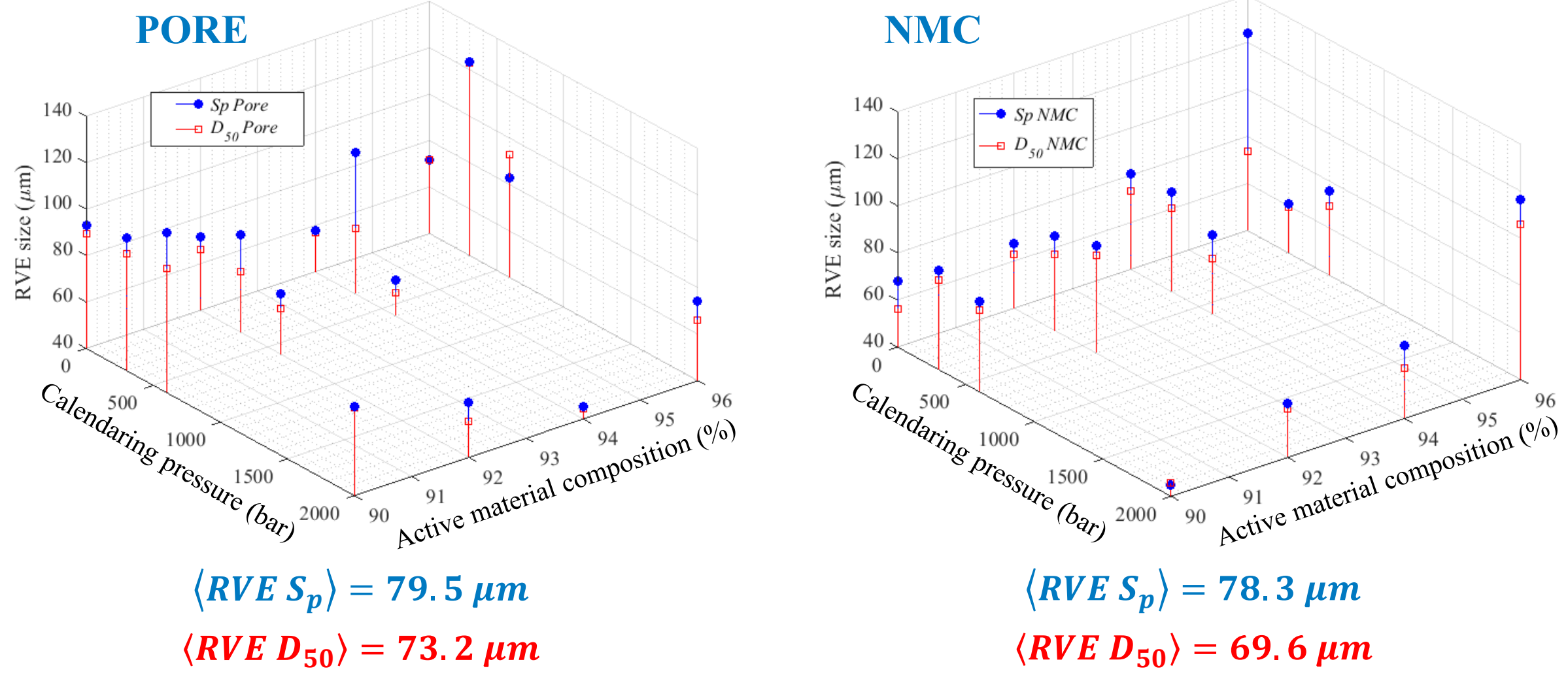

As expected, Representative Volume Element sizes are very similar between $S_{p}$ and $D_{50}$

NMC: $\left\langle R V E S_{p}\right\rangle \sim 1.12 \times\left\langle R V E D_{50}\right\rangle$

Pore: $\left\langle R V E S_{p}\right\rangle \sim 1.09 \times\left\langle R V E D_{50}\right\rangle$ 


\section{Annexes: Particle size}




\section{Particle size: statistical approach}

T. Kanit et al., International Journal of Solids and Structures 40 (2003) 3647-3679

$$
\begin{aligned}
& C(V, \bar{h})\left\{\begin{array}{l}
=P\left\{(x, y, z) \epsilon \mathrm{V} \cap\left(x+h_{1}, y+h_{2}, z+h_{3}\right) \in \mathrm{V}\right\} \\
=\frac{1}{N} \times \sum_{i=1}^{N} k(x, y, z) \times k\left(x+h_{1}, y+h_{2}, z+h_{3}\right)
\end{array}\right. \\
& \text { with }\left\{\begin{array}{c}
k(x, y, z)=\left\{\begin{array}{l}
1 \text { if }(x, y, z) \in V \\
0 \text { if }(x, y, z) \notin V
\end{array}\right. \\
\bar{h}=h_{1} \overline{e_{x}}+h_{2} \overline{e_{y}}+h_{3} \overline{e_{z}}
\end{array}\right.
\end{aligned}
$$

\section{Using the conditional probability:}

(a)

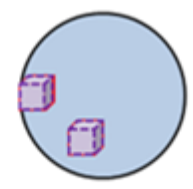

(b)

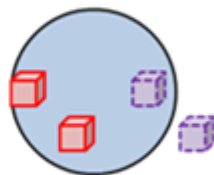

(c)

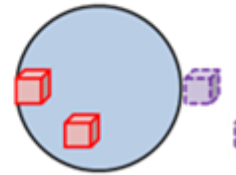

$h=0$

Maximum correlation: $P\{x+\bar{h} \in V \mid x \in V\}=1$

$h<D$

Partial correlation:

$P\{x \in V\}<P\{x+\bar{h} \in V \mid x \in V\}<1$

$h \geq D$

Zero correlation:

$P\{x+\bar{h} \in V \mid x \in V\}=P\{x+\bar{h} \in V\}$

$$
C(V, \bar{h})=P\{\bar{x}+\bar{h} \in V \mid \bar{x} \in V\} \times P\{\bar{x} \in V\}
$$

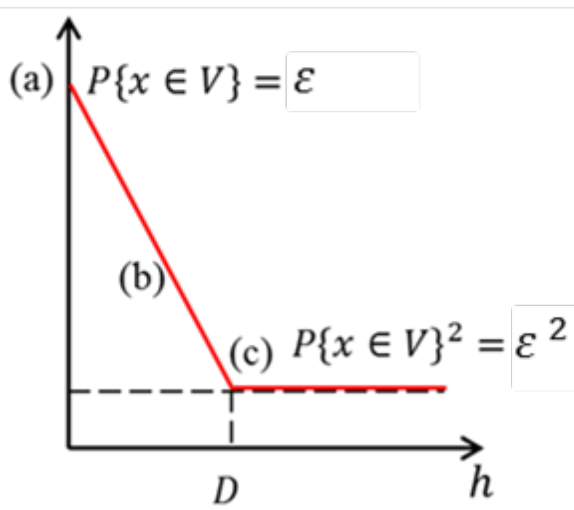

The zero correlation (stage $\mathrm{c}$ in the figure) is reached for different value of $\mathrm{h}$ for particles of different size. The total loss of correlation (and thus the asymptotic value $\varepsilon^{2}$ ) is only achieved when all voxels have lost their correlation from their initial position: thus it indicates the largest particle size and not the mean particle size. 


\section{Particle size: statistical approach}

Original

addition to the method

\section{Using the conditional probability:}

$$
\begin{gathered}
C(V, \bar{h})=P\{\bar{x}+\bar{h} \in V \mid \bar{x} \in V\} \times P\{\bar{x} \in V\} \\
P\{x+\bar{h} \in V \mid x \in V\}=P\{A\}+P\{B\}
\end{gathered}
$$

with $\left\{\begin{array}{c}\{A\}=\{x+\bar{h} \text { belongs to the initial sphere on which } x \text { belongs }\} \\ \{B\}=\{x+\bar{h} \text { belongs to another sphere of same diameter }\}\end{array}\right.$

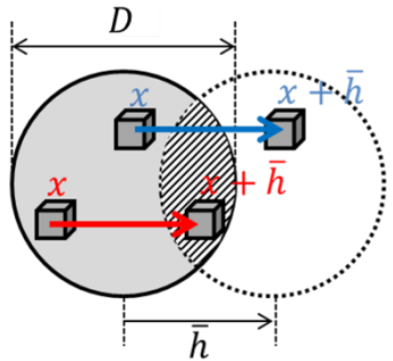

(S)

Events $\mathrm{A}$ and $\mathrm{B}$ are contained within the volume $V_{S}$ of a sphere of diameter $D$. The ratio of voxels $x+\bar{h}$ that will be tested for the event $\mathrm{A}$ is $V_{I S} / V_{S}$, and it is sure they all belong to the phase $V$ (since they are still in the initial particle). The ratio of voxels $x+\bar{h}$ that will be tested for the event B is $\left(V_{S}-V_{I S}\right) / V_{S}$ and the probability they belong to the phase $V$ is the volume fraction of $V$ (since they are outside the initial particle). Then:

$$
P\{A\}=\frac{V_{I S}}{V_{S}} \times 1
$$

$P\{B\}=\left(\frac{V_{S}-V_{I S}}{V_{S}}\right) \times \varepsilon$

Fitting the diameter $\mathrm{h}$ of the theoretical

covariance

$$
V_{S}=\frac{\pi}{6} \times D^{3}
$$

0 otherwise

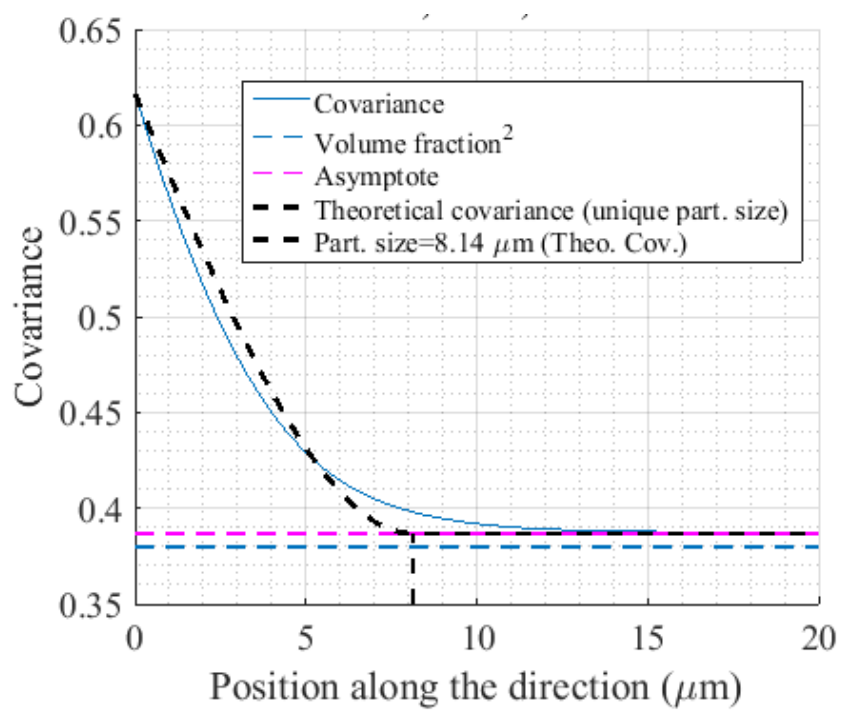




\section{Particle size: watershed method}

Luc Vincent and Pierre Soille, IEEE Transactions on pattern analysis and machine intelligence, Vol 13, No 6, 1991

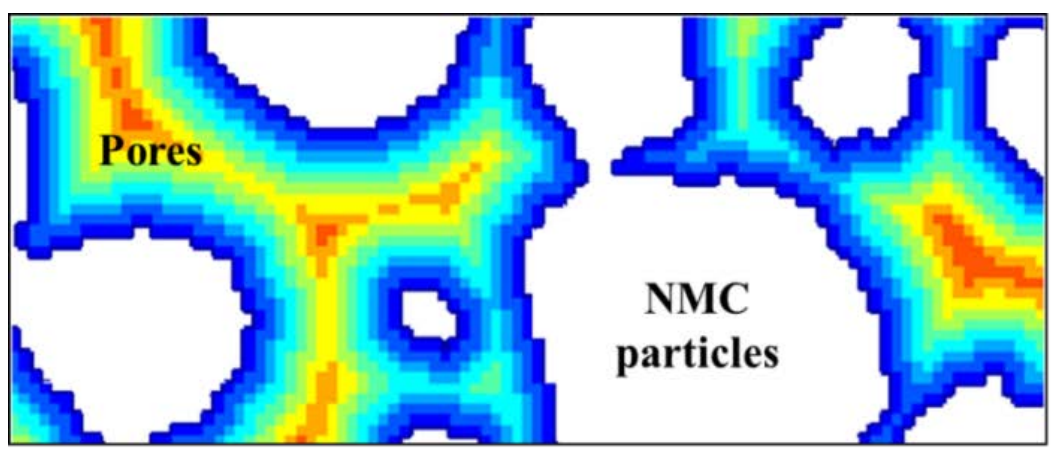

(a)
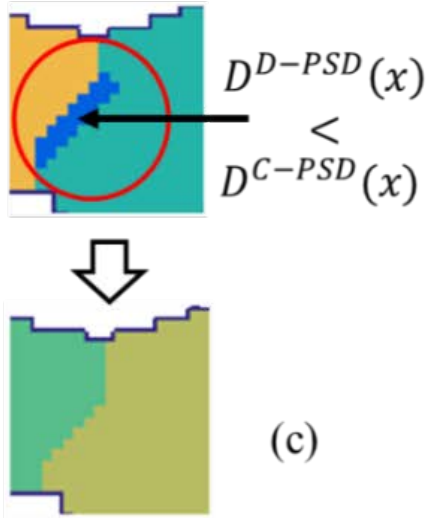

(c)

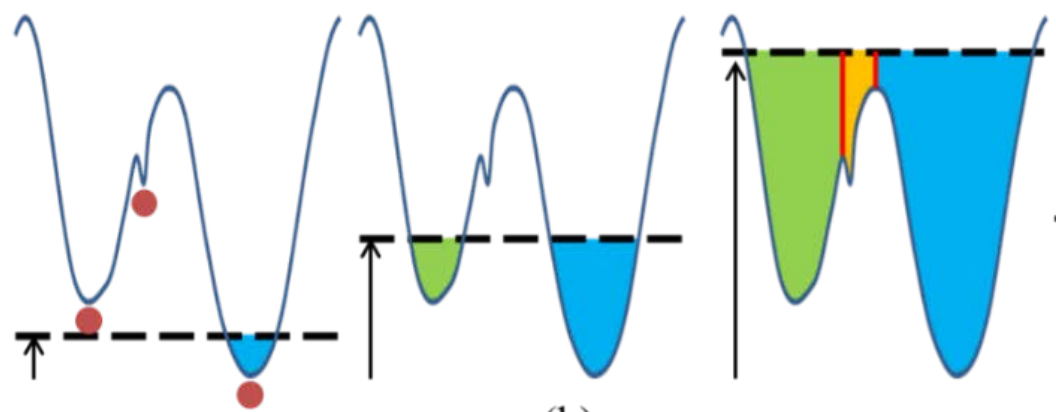

- Water sources

$\pi^{-}$Water level

I Imaginary dam

(b)

(a) Euclidean distance transform (EDT) of the pores calculated on a two-dimensional subset of a positive electrode. Values are scaled with the color: from dark blue to red (maximum distance). (b) One-dimensional schematic of the immersion watershed process, from left to right. Three catchment basins (i.e. distinct particles) have been identified. The smallest one appears to be an artifact due to the local noise gradient. (c) Original addition to the algorithm: Voxels in the smallest distinct particle are considered to be irrelevant since their local size verifies $D^{D-P S D}(x)<D^{C-P S D}(x)$. The red circle illustrates $D^{C-P S D}(x)$ while one unique color is attributed to $D^{D-P S D}(x)$. 


\section{Particle size: watershed method}

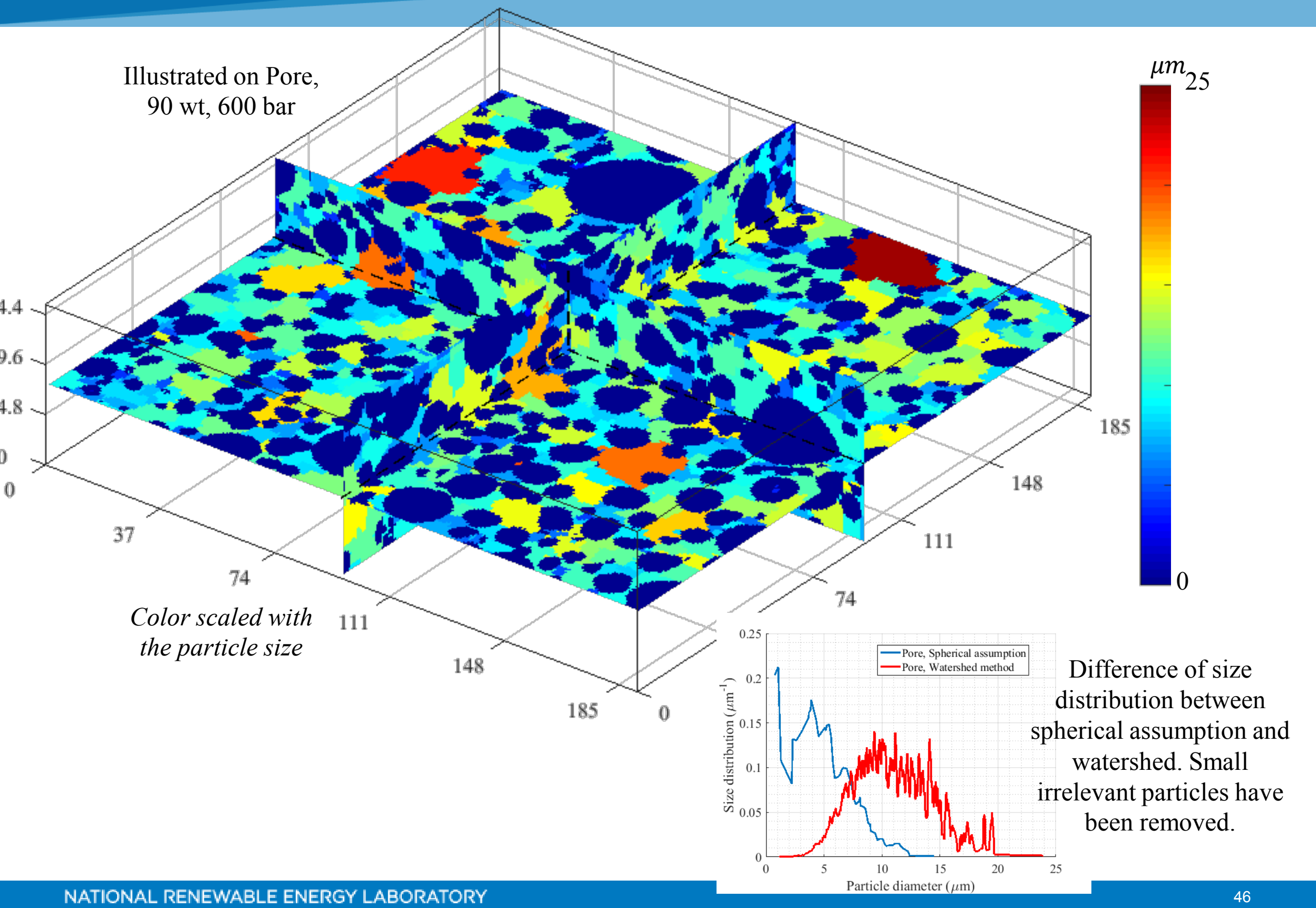




\section{Annexes: Tortuosity}

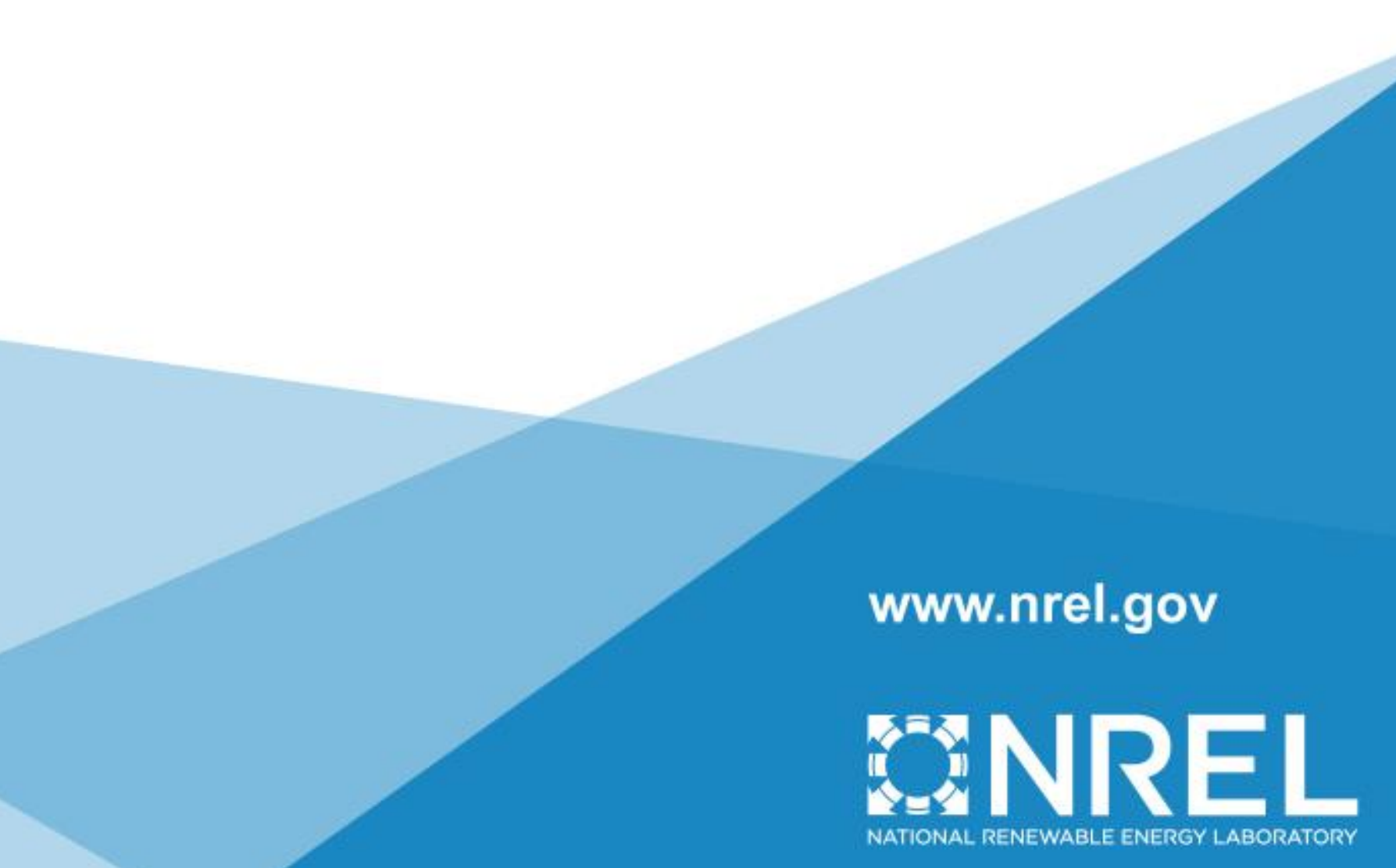




\section{Factor of tortuosity}

\section{- Factor of tortuosity (homogenization method)}

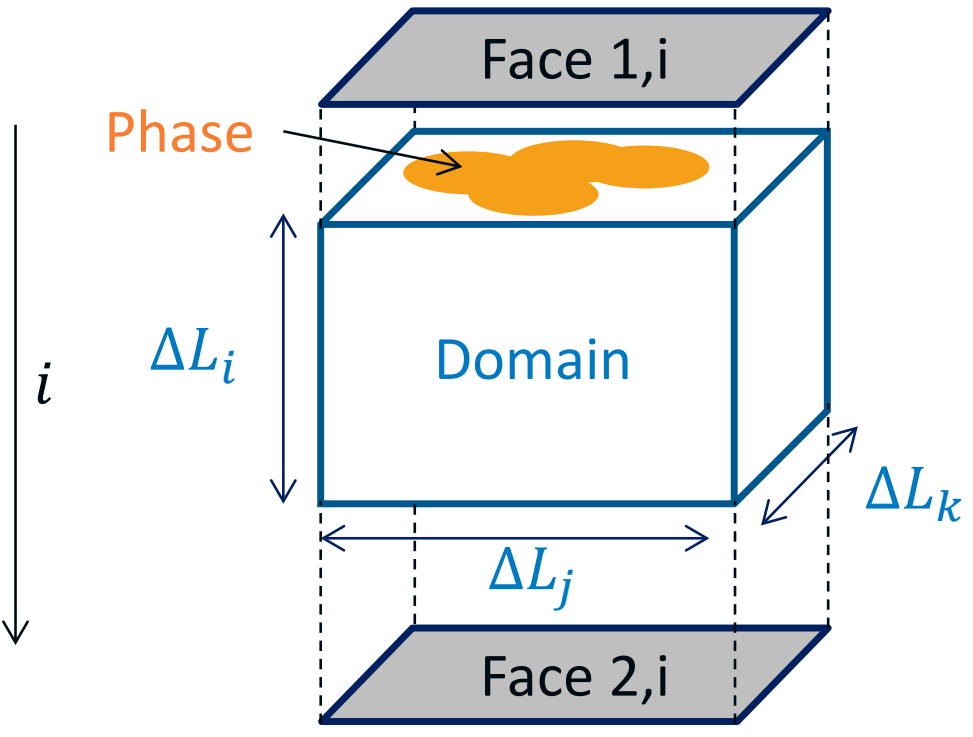

Effective section area normal to direction $i$ :

$$
S_{i}^{e f f}=\Delta L_{j} \times \Delta L_{k}
$$

Actual section area normal to direction $i$ at face 1: $S_{i, f a c e}^{\text {actual }}=\iint_{F_{1, i}} 1 d s($ phase $)$
Step 1: Mean concentration at the face $F_{1}$

$$
c_{i, 1}=\iint_{F_{1, i}} c d s / S_{i, \text { face } 1}^{\text {actual }}
$$

Step 2: Effective surface density flux at the face $F_{1}$

$$
\varphi_{i, 1}=\iint_{F_{i, 1}}-k_{b u l k} \times \overline{\operatorname{grad}}(c) \cdot \bar{n} d s / S_{i}^{e f f}
$$

Step 3: Deduce the effective diffusion coefficient (1D diffusion law)

$$
k_{i}^{e f f}=-\varphi_{i, 1} \times \frac{\Delta L_{i}}{c_{i, 2}-c_{i, 1}}
$$

Step 4: Deduce the factor of tortuosity

$$
\tau_{i}=\varepsilon \times k_{\text {bulk }} / k_{i}^{\text {eff }} \geq 1
$$




\section{Factor of tortuosity: Representative volume element analysis}

- RVE analysis on $\boldsymbol{\tau}_{\text {through-plane dir. }}$ with different boundary conditions
For which domain's size

$\tau_{\text {through-plane dir. }}$ does not depend on the chosen $B C$ ?

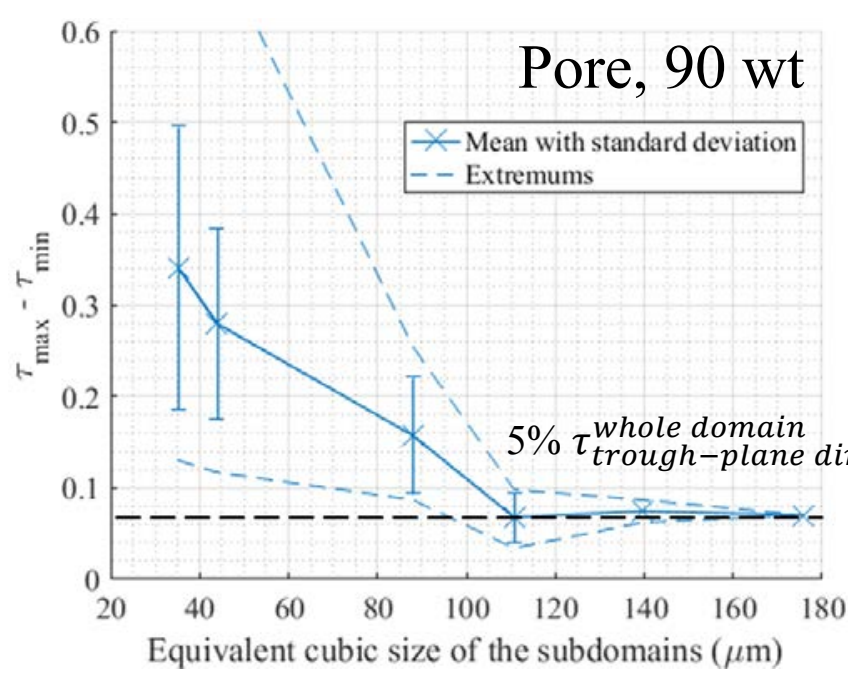

Equivalent cubic size of the subdomains $(\mu \mathrm{m})$

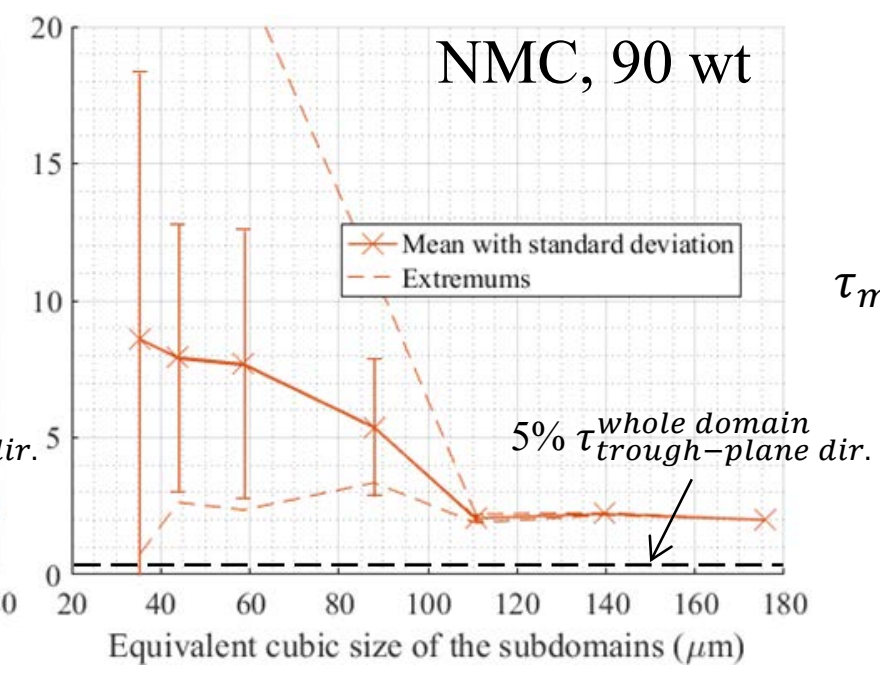

Equivalent cubic size of the subdomains $(\mu \mathrm{m})$
RVE criterion is different:

Instead of std must $\rightarrow 0$ :

$\boldsymbol{\tau}_{\text {max }}-\boldsymbol{\tau}_{\min }$ must $\rightarrow \mathbf{0}$

$\tau_{\text {max }}-\tau_{\min } \leq 5 \%$ of $\tau_{\text {through-plane dir. }}^{\text {whole domain }}$

To remove the boundary conditions dependence, a larger domain is required.
Tortuosity Boundary conditions table (written in brackets are the RVE)

\begin{tabular}{l|c:c|c:c}
\hline & \multicolumn{2}{|c|}{ Pore } & \multicolumn{2}{c}{ NMC } \\
\hline \multirow{2}{*}{$\tau_{\max }-\tau_{\min }$} & $\mathrm{UC}$ & $\mathrm{C}(2000 \mathrm{bar})$ & $\mathrm{UC}$ & $\mathrm{C}(2000 \mathrm{bar})$ \\
& $(110.8 \mu \mathrm{m})$ & $(115.5 \mu \mathrm{m})$ & $(>175 \mu \mathrm{m})$ & $(>183 \mu \mathrm{m})$ \\
\hline
\end{tabular}

RVE $\tau_{\text {Boundary conditions }}>$ RVE $\tau_{\text {Dirichlet-Neumann }}^{\text {Through-plane dir. }}$
The plateau obtained for large domains indicates a convergence issue. It could be solved with a quadratic interpolation (better convergence is expected).

T. Kanit et al., Inter. J. of Solids and Structures, 40 (2003) 3647-3679

J. Laurencin et al., JPS, 198 (2012) 182-189 


\section{Factor of tortuosity: rule of mixture \& percolation loss}

\section{Why factor of tortuosity obtained on subdomains differs from the whole domain?}

Loss of percolation on small subdomains should increase tortuosity by creating local bottleneck at the edges... but we see the opposite.

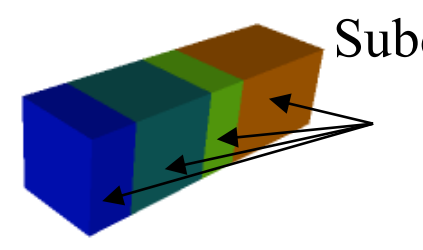

diffusion coefficients

$$
\left.\left.k_{i} \in\right] 0 ; 1\right]
$$

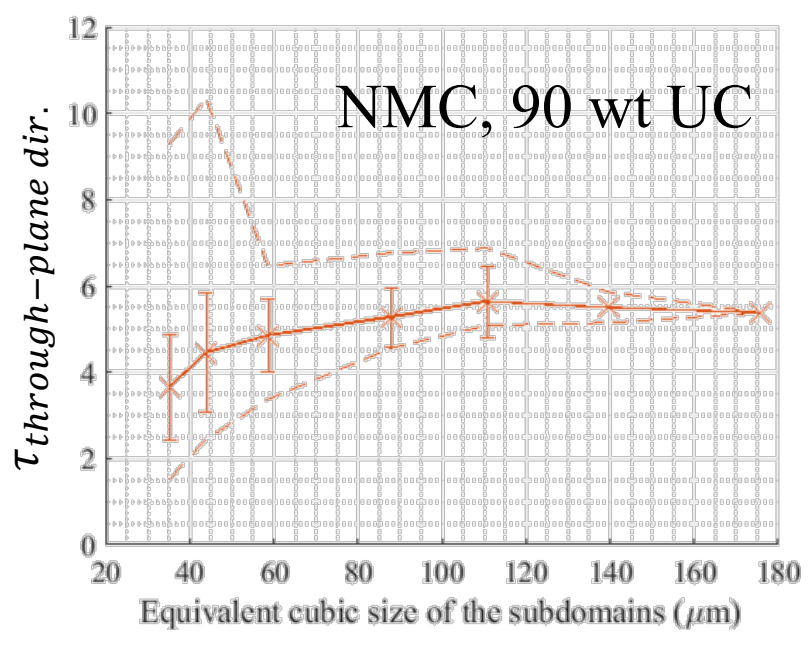

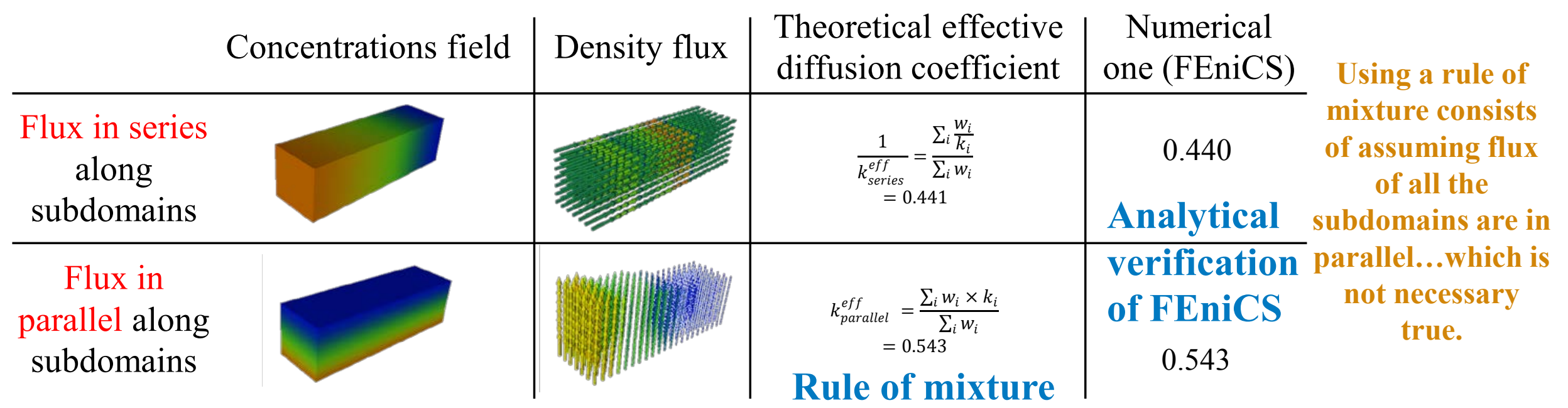

